\title{
ON THE STABILITY OF RACING SAILING BOATS WITH FOILS
}

\author{
PHILIPPE DESTUYNDER* AND CAROLINE FABRE** \\ * Département d'ingénierie mathématique, laboratoire M2N \\ Conservatoire National des Arts et Métiers \\ 292, rue saint Martin, 75003 Paris France \\ philippe.destuynder@cnam.fr \\ ** Laboratoire de mathématiques d'Orsay, UMR 8628 \\ Univ Paris-Sud, CNRS, Université Paris-Saclay \\ Orsay 91405 France \\ caroline.fabre@u-psud.fr
}

ABSTRACT. The development of foils for racing boats has changed the strategy of sailing. Recently, the America's cup held in San Francisco, has been the theatre of a tragicomic history due to the foils. During the last round, the New-Zealand boat was winning by 8 to 1 against the defender USA. The winner is the first with 9 victories. USA team understood suddenly (may be) how to use the control of the pitching of the main foils by adjusting the rake in order to stabilize the ship. And USA won by 9 victories against 8 to the challenger NZ. Our goal in this paper is to point out few aspects which could be taken into account in order to improve this mysterious control law which is known as the key of the victory of the USA team. There are certainly many reasons and in particular the cleverness of the sailors and of all the engineering team behind this project. But it appears interesting to have a mathematical discussion, even if it is a partial one, on the mechanical behaviour of these extraordinary sailing boats. The numerical examples given here are not the true ones. They have just been invented in order to explain the theoretical developments concerning three points: the possibility of tacking on the foils for sailing upwind, the nature of foiling instabilities, if there are, when the boat is flying and the control laws.

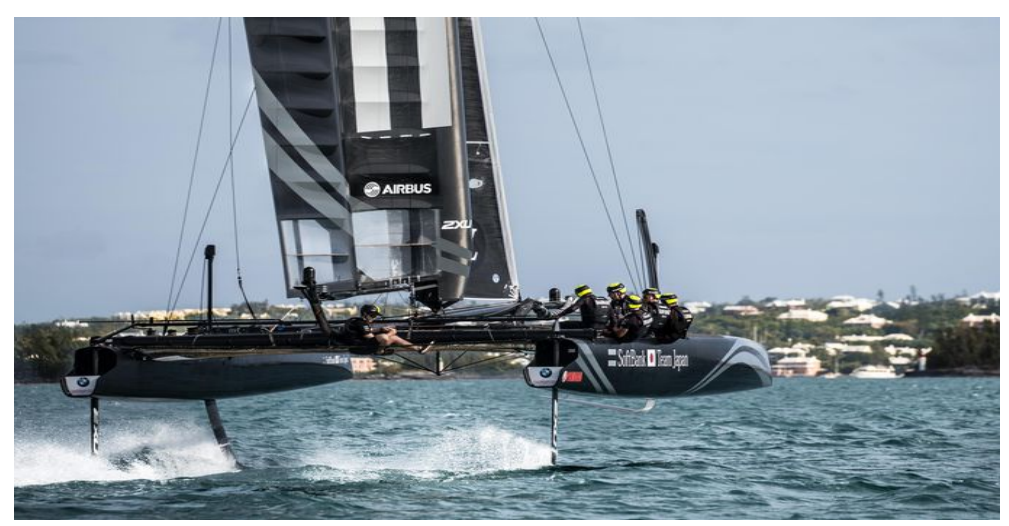

FIGURE 1. Principle of the flying boat with the AC45 of Oracle USA Team - TV snapshot

Date: 03-26-2016.

2010 Mathematics Subject Classification. Primary: 35C07, 65M15; Secondary: 35M12, 65T60.

Key words and phrases. hydrodynamics of foils, mathematical analysis, stability . 
PH. D.

1. Introduction. The control of foiling during the America's cup appeared to be a determinant point in the success of Oracle Team USA (OTUSA). In particular during upwind legs, when the boat had to avoid the waves created by the wake of the preceding boat, the automatic stabilization is a fundamental advantage that OTUSA exploited in a smart way and finally won the competition. Such situations are represented on the figures 2 taken from TV transmissions during the America's cup in San Francisco (September 2013).
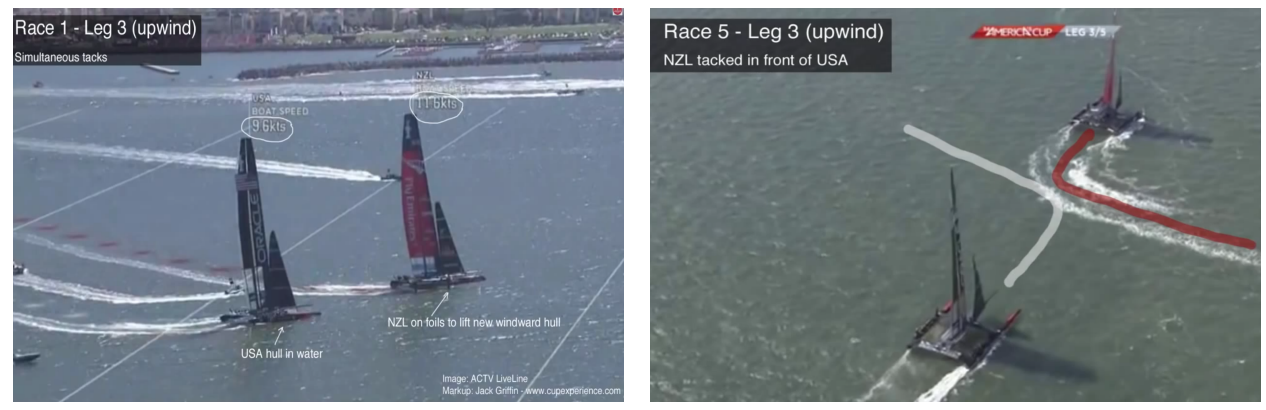

FIGURE 2. Two situations where the controlled foiling could avoid to tack and to loose the race

In this paper, we have tried to give a simple and precise mathematical model and analysis of such boats. Even if a more industrial 3D analysis would be obviously more realistic, and in order to be as simple as possible, we restrict our analysis to a bi-dimensional case. Hence, only two movements of the ship are taken into account: the heaving which is a normal displacement to the surface of the sea, and the pitching which is the rotation around a horizontal axis transverse to the main direction of the ship. Hence, the yawing angle and the rolling are eliminated from our model. Obviously they are meaningful, but according to our mind, not necessary for the understanding of our purpose.

The inclination of the main foil should be manually driven but a hydraulic ram can be used for the control process (rules of the race) using the high pressure collected from a small hole in the foil. Because the system is a second order one (with inertia, damping and stiffness), only a phase control can lead to optimal results. This driven angle is named the rake. It appears, in the numerical simulations, that the regulation law strongly depends on the ship velocity. Even if the experimental data that we introduce in our numerical model could be improved, they are sufficient in order to give an idea of how things work.

The aim of this paper is to obtain a faithful dynamical model of the movement of the boat which respects the following facts :

- the existence of the foiling velocity under which the boat can't stand up on its foil

- the possibility that the velocity of the boat can be greater than the wind velocity,

- the possibility to discuss the stall flutter phenomenon of the foils.

The plan of our work is the following one: we first compute the aerodynamic propulsion force due to the wind and which is applied on the sails bearing in mind its importance since it is the unique energy source of the boat. We then establish a nonlinear model which take into account the apparent velocity of the water flow at the rear and main foils. We then analyze the steady state of the non linear model and a numerical study will point out the overspeed phenomenae. We finally study the stability of the linear model and discuss the stall-flutter phenomenon. A part of the work was the characterization of the foiling velocity at which the boat takes off. All our theoritical work is illustrated with numerical 
results with computations performed with Matlab. The fundamental tools for the numerical analysis are developped in the book of P.-G. Ciarlet [4].

\section{Dynamical model of the boat.}

2.1. Description of the boat and main notations. The orthonormal basis of $\mathbb{R}^{3}$ is denoted by $\left(e_{x}, e_{y}, e_{z}\right)$. The velocity of the boat is $-u e_{x}$ where $u>0$ and therefore the velocity of the flow of the water in the basis connected to the boat is $u e_{x}$. As said before, the movement is assumed to be represented by two functions (see figure 3): the heaving $z$ and the pitching angle $\gamma$ in the plan $\left(e_{z}, e_{x}\right)$. The equilibrium is written at an arbitrary point - say $O$. For sake of convenience, it is chosen to be the center of rotation of the main foil.

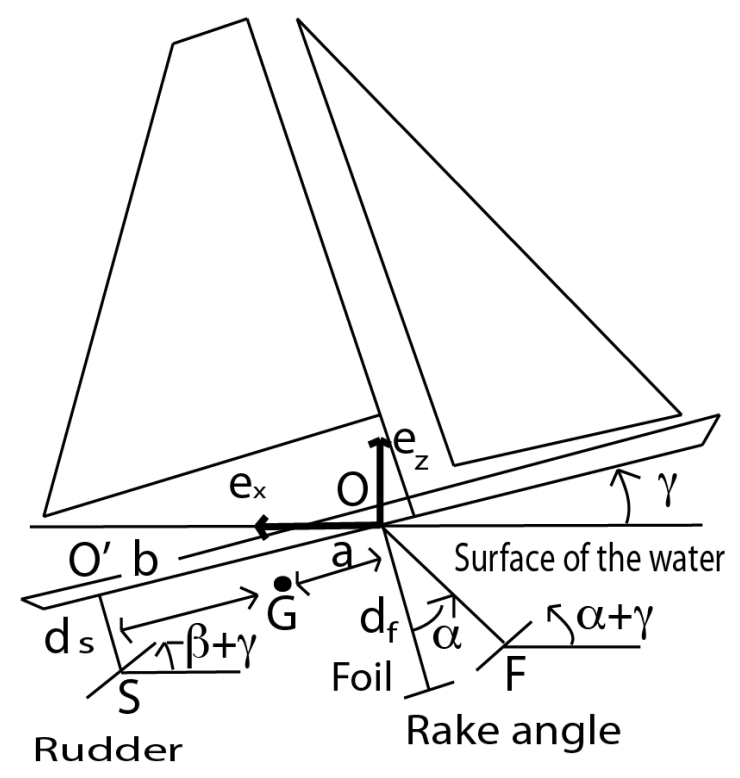

FIGURE 3. The boat with the foils

The following notations are used:

\section{Characteristics of the boat, of the air and of the water:}

$\diamond \varrho_{a}$ mass density of the air,

$\diamond \varrho_{e}$ mass density of the water,

$\diamond g=9.81 \mathrm{~m} / \mathrm{s}^{2}$ is the gravity,

$\diamond-u e_{x}$ velocity of the ship,

$\diamond M$ is the mass of the ship,

$\diamond G$ center of mass of the boat,

$\diamond J_{G}$ is the inertia around the center of mass $G$ in the pitching,

$\diamond J_{O}$ is the inertia around the center of mass $O$ in the pitching,

$\diamond M_{o}$ is the moment of the external forces at point $O$ in the pitching,

$\diamond d_{s}=O^{\prime} S$ is the length of the stick supporting the steering rudder, $O^{\prime}$ being the anchor point of the rear foil;

$\diamond d_{f}=O F$ is the length of the foil in the depth direction;

$\diamond S_{s}, S_{f}$ are respectively the cross sections of the foils at the extremities of the rudder and the main foil; 
$\diamond a\left(\right.$ respectively $b$ ) is the distance between the center of mass and $O$ (respectively $O^{\prime}$ )

$\diamond h=a+b=O O^{\prime}$

$\diamond d_{o g}$ is the distance from the rotation point of the foil to the center of mass of this foil

Variables for the description of the movement of the boat:

$\diamond z$ is the heaving

$\diamond \gamma$ is the pitching angle

For the angles, apparent velocities and forces:

$\diamond \alpha$ is the angle of attack of the mail foil,

$\diamond \beta$ is the angle of attack of the rear foil, it is supposed to be fixed,

$\diamond c_{z f}$ and $c_{z s}$ are the lift hydrodynamic coefficients for the main foil and the rear foil. They are continuous in their variables,

$\diamond c_{m f}$ et $c_{m s}$ are the pitching hydrodynamic coefficients at points $F$ and $S$. They are continuous functions in their variables,

$\diamond v$ is the absolute wind velocity: it is in the plane $\left(e_{x}, e_{y}\right)$,

$\diamond V$ the modulus of $v$, absolute wind velocity,

$\diamond \theta=\left(\widehat{v,-e_{x}}\right)$ is the angle between the velocity of the wind and the direction in which the boat is moving forward,

$\diamond V_{a}$ is the modulus of the apparent velocity of the wind,

$\diamond V_{a s}, V_{a f}$ are respectively the apparent flow velocity at the two foils: one on the rudder and the other one which, is the main one, supported by the daggerboard;

$\diamond u$ is the modulus of the velocity of the ship,

$\diamond c_{f}$ and $\xi$ are respectively the stiffness and the damping coefficient of the system used for the stabilisation of the main foil.

The forces applied to the ship and implying an evolution of these two previous functions, are those due the rear and the main foils. The local hydrodynamical coefficients $\left(c_{z}\right.$ for the lift and $c_{m}$ for the pitching moment) depend respectively on the apparent local angle of attack of each foil. For the rear foil, it is denoted by $(\beta+\gamma)_{a}$ and $(\alpha+\gamma)_{a}$ for the main one.

2.2. The apparent velocity of the wind. Even if it is a side subject for our main purpose, it is worth to recall how the apparent wind velocity can induce overspeed for particular positions of the boat with respect to the direction of the wind. The formulae used in this section, aren't original. Our goal is only to show with a simple numerical simulation, the influence of various parameters on the boat velocity and mainly the one of the sailing position and of the drag coefficient of the bows or the foils in the water.

Let us consider the situation represented on figure ??. Following the notations of this figure, the apparent wind velocity is given by:

$$
v_{a}=v-u e_{x}=-(V \cos (\theta)+u) e_{x}+V \sin (\theta) e_{y},
$$

and its modules is equal to:

$$
V_{a}^{2}=V^{2}\left[1+\frac{u^{2}}{V^{2}}+2 \frac{u}{V} \cos (\theta)\right] .
$$

The apparent angle between the normal to the sail plane and this apparent wind velocity is $\mu_{a}=\widehat{\left(n, v_{a}\right)}$ and it satisfies:

$$
v_{a}=V_{a} \cos \left(\frac{\pi}{2}+\mu_{a}\right) \tau+V_{a} \sin \left(\frac{\pi}{2}+\mu_{a}\right) n=V_{a}\left[\cos \left(\mu_{a}\right) n-\sin \left(\mu_{a}\right) \tau\right] .
$$




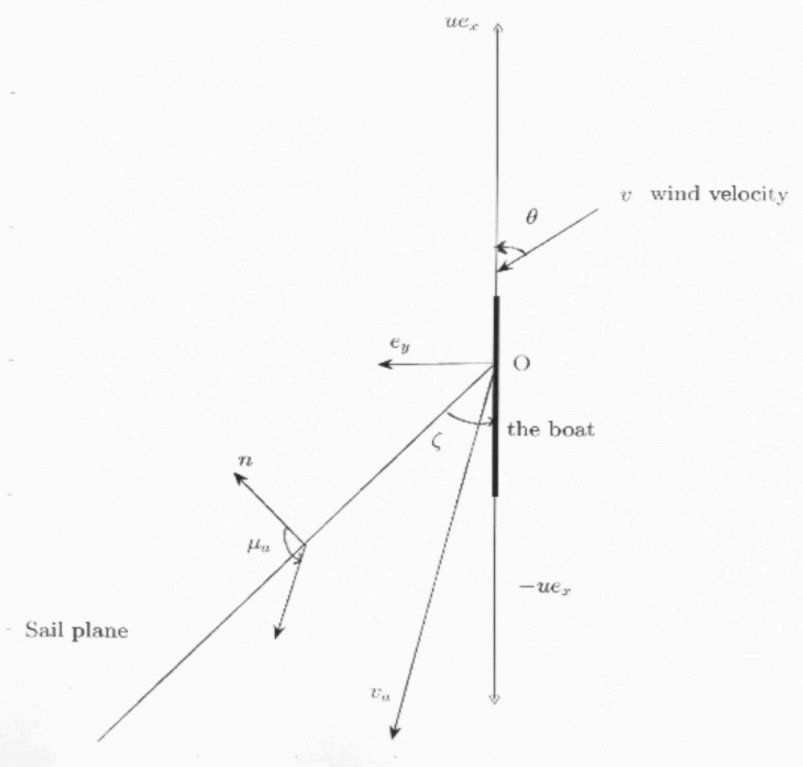

FIGURE 4. The sail plane and the apparent wind

and therefore:

$$
\begin{aligned}
v_{a} \cdot n & =V_{a} \cos \left(\mu_{a}\right)=\left(v-u e_{x}\right) \cdot n=v \cdot n-\sin (\zeta) u \\
& =-V \cos (\theta) \sin (\zeta)+V \sin (\theta) \cos (\zeta)-\sin (\zeta) u \\
& =V \sin (\theta-\zeta)-\sin (\zeta) u .
\end{aligned}
$$

Using (3), we get:

$$
V_{a} \cos \left(\mu_{a}\right)=V \sin (\theta-\zeta)-\sin (\zeta) u,
$$

and thus:

$$
\mu_{a}=\arccos \left(\frac{\sin (\theta-\zeta)-\frac{u}{V} \sin (\zeta)}{\sqrt{1+\frac{u^{2}}{V^{2}}+2 \frac{u}{V} \cos (\theta)}}\right) .
$$

The propulsion force due to the wind denoted by $F_{x}$, is the projection on the direction $e_{x}$ of the aerodynamical force applied to the sail. For sake of simplicity it can be written (the square of the $\cos \left(\mu_{a}\right)$ takes into account the normal component of the apparent wind velocity and $n$ is the unit normal to the sail plane):

$$
F_{x}=\frac{1}{2} \rho_{a} S_{a}\left(v_{a} . n\right)^{2} c_{x a} n . e_{x} .
$$

In fact, a correction coefficient is included in the surface $S_{a}$ which takes into account the aerodynamical coefficient $c_{z}\left(\mu_{a}\right)$ of the sail. With (3), we get:

$$
F_{x}=\frac{1}{2} \rho_{a} S_{a} V_{a}^{2} \cos ^{2}\left(\mu_{a}\right) c_{x a} \sin (\zeta) .
$$

The drag force is the sum of two contributions: one due to the sail and another one due to the drag in the water of the bows (zero during the flight) and the foils which are always 
immersed. Furthermore, the last term depends on both $\beta+\gamma$ and $\alpha+\gamma$. Let us assume that this drag force can be evaluated by:

$$
T_{x}=\frac{1}{2} \rho_{e} S_{e} u^{2} c_{x e},
$$

where $S_{e}$ is the cross section immerged into the water and $c_{x e}$ the corresponding drag coefficient. In fact, the important number is the product $S_{e} c_{x e}$. It is about $1.5 \mathrm{~m}^{2}$ for a ship floating and about $.1 \mathrm{~m}^{2}$ for a flying one as far as the profil of the foils are correctly drawn.

2.3. The overspeed phenomenon. We deduce that the velocity $u$ of the boat is obtained by solving the equation:

$$
F_{x}-T_{x}=0 .
$$

Due to the complexity of this equation, it is easier to perform numerical tests. We have drawn on Figure 5 the sign of the function $F_{x}-T_{x}$ with respect to the two variables $u$ on the abscissa which is the velocity of the ship and $\zeta$ on the ordinate which is the angle of the sail plane with the direction $e_{x}$ (velocity of the boat). The boundary between the two areas (black and white) are the solutions.

The Figure $5 \mathrm{a}$ is concerned with a floating boat (which have its bows in the water) whereas Figure $5 \mathrm{~b}$ and Figure 5c illustrate the case of a flying boat.

For the floating boat, it appears, with the set of data used, that the absolute wind velocity can't be overtaken with our choice for the physical data. For the flying boat, the absolute wind velocity can be exceeded. This is due to the reduction of the drag force on the bows in the water. In fact the pictures on these figures show that a tacking for upwind sailing is much better with large angles concerning the velocity because it enables to make the boat flying above the water using the foils. And even if the distance covered is more important, the time necessary can be smaller. But the flight must be stabilized similarly to what is done with an aircraft. Because, even if the flight is stable, there can be perturbations due to the gravity waves for instance. This is more critical if the boat has to cross over the wake of a preceding boat. In fact the phenomenae are very close for a simple reason: the ratio between the aerodynamical forces on the wing of an aircraft is similar to the one applied to the foil of a flying ship. In fact, the ratio between the mass density of the water and the one of the air is about $1000 / 1.2 \simeq 833$ and the one between the square of the velocities is about $(10 / 290)^{2} \simeq 1 / 841$.

And the equivalence is deduced from the fact that the forces are proportional to the mass density times the square of the velocity.

\section{Dynamical equations.}

3.1. The apparent flow velocities on the foils. The apparent velocity of the water on the foils implies the terms $\dot{\gamma}$ and $\dot{z}$. It is the difference between the wind velocity and the one of the boat. First of all, let us give the expressions of the velocities of the points $S$ and $F$ corresponding respectively to the rudder and the main foil where the hydodynamic forces are given from hydrotunnel tests. We refer to Figure 3.

One has:

$$
V_{s}=(\dot{z}-h \dot{\gamma} \cos (\gamma)) e_{z}-h \dot{\gamma} \sin (\gamma) e_{x}+\frac{d \overrightarrow{O^{\prime} S}}{d t} .
$$

On another way, we have:

$$
\begin{aligned}
\overrightarrow{O^{\prime} S} & =d_{s} \cos \left(\gamma-\frac{\pi}{2}\right)\left(-e_{x}\right)+d_{s} \sin \left(\gamma-\frac{\pi}{2}\right) e_{z} \\
& =-d_{s} \sin (\gamma) e_{x}-d_{s} \cos (\gamma) e_{z},
\end{aligned}
$$


A floating boat: $S C_{x e}=0.3 \mathrm{~m}^{2}, V=20 \mathrm{~m} / \mathrm{s}$

$\theta=0.67 r d$ (left) and $\theta=1.03 r d$ (right)
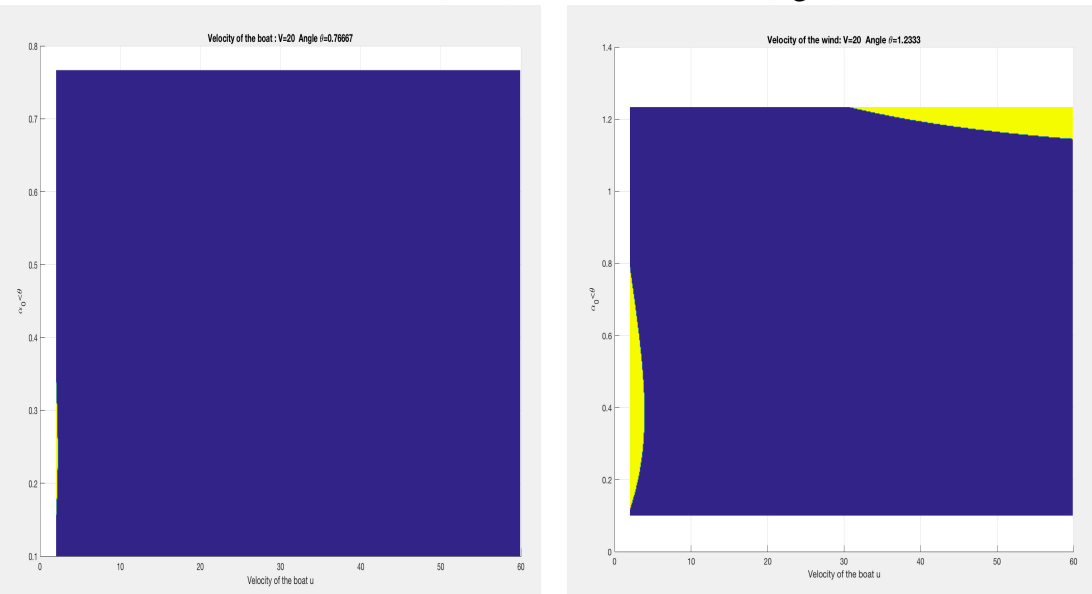

Figure 5a. The speed is smaller than $V$.

A flying boat: $S C X_{e}=0.01 \mathrm{~m}^{2}, V=10 \mathrm{~m} / \mathrm{s}$

$\theta=0.67 r d$ (left) and $\theta=1.03 r d$ (right)
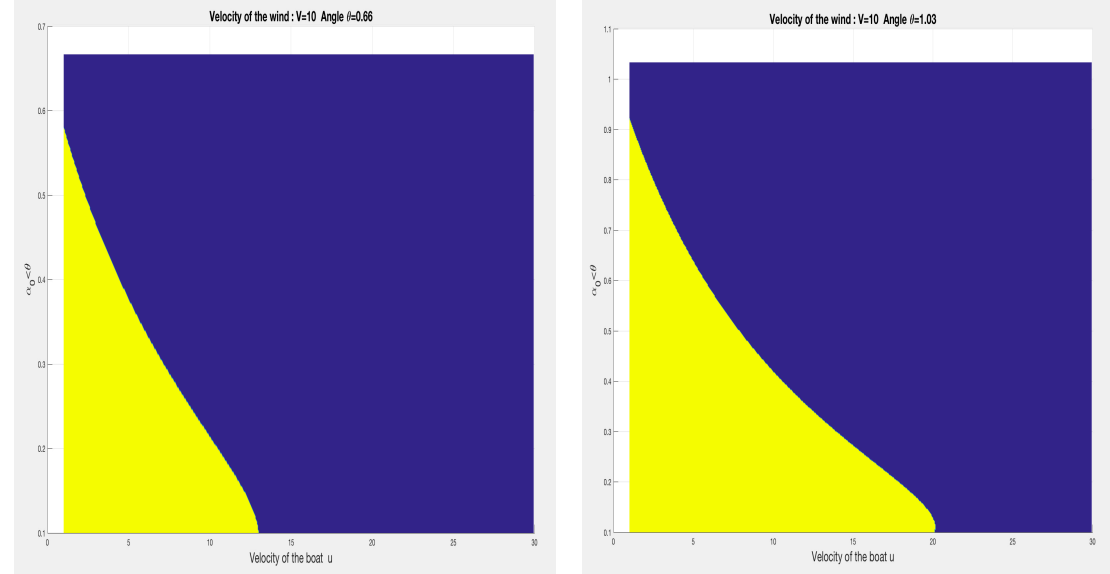

Figure $5 \mathrm{~b}$. The speed can be greater than $2 \mathrm{~V}$.

A flying boat: $S C X_{e}=0.01 \mathrm{~m}^{2}, V=20 \mathrm{~m} / \mathrm{s}$
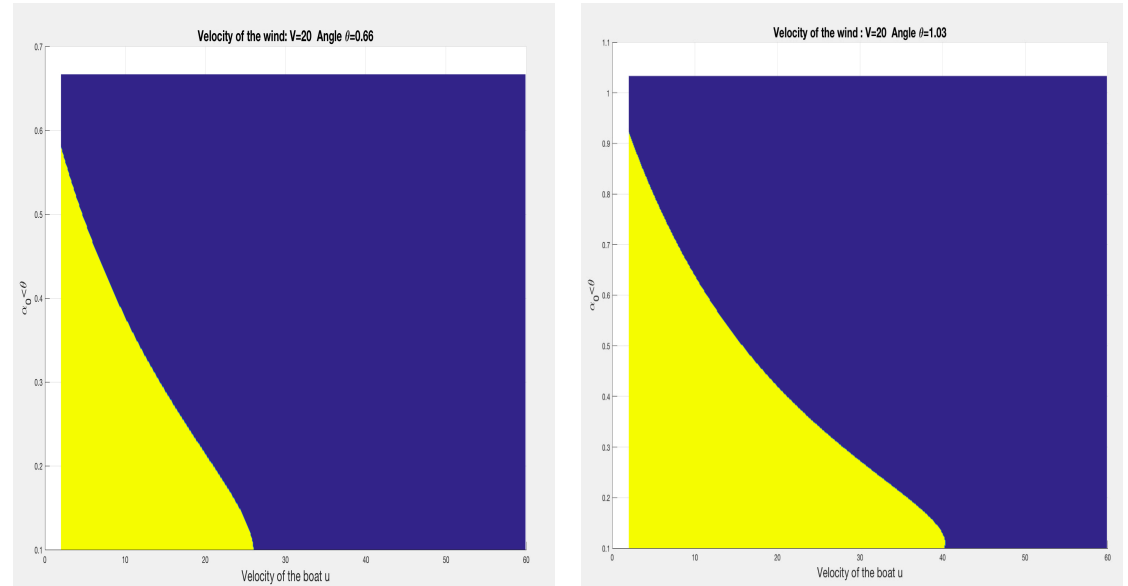

Figure $5 \mathrm{c}$. The speed can be greater than $2 \mathrm{~V}$.

FIGURE 5. 
and therefore the velocity of the flow at point $S$ is equal to:

$$
V_{s}=\left(\dot{z}-h \dot{\gamma} \cos (\gamma)+d_{s} \dot{\gamma} \sin (\gamma)\right) e_{z}+\left(-h \dot{\gamma} \sin (\gamma)-d_{s} \dot{\gamma} \cos (\gamma)\right) e_{x}
$$

We get analogous results at point $F$ where the velocity of the flow is:

$$
V_{f}=\left(\dot{z}+d_{f}(\dot{\alpha}+\dot{\gamma}) \sin (\alpha+\gamma)\right) e_{z}-d_{f}(\dot{\alpha}+\dot{\gamma}) \cos (\gamma) e_{x} .
$$

The computations of the hydrodynamic apparent velocities are performed at points $S$ et $F$ in the axis $\left(e_{x}, e_{z}\right)$. These apparent flow velocities are defined as the difference between the absolute flow velocity and the one of the point considered. Let us notice that there are two different notions for the apparent velocity: the one of the wind and the one of the hydrodynamic flow on the foils. From now on, it is the second one which is taken under consideration. It is given by the following formulae where $V$ is therefore the apparent flow velocity:

$$
V_{a s}=u e_{x}-V_{s} \quad \text { et } \quad V_{a f}=u e_{x}-V_{f}
$$

and we then obtain:

$$
V_{a s}=\left(u+\dot{\gamma}\left(h \sin (\gamma)+d_{s} \cos (\gamma)\right)\right) e_{x}-\left(\dot{z}-\dot{\gamma}\left(h \cos (\gamma)-d_{s} \sin (\gamma)\right)\right) e_{z}
$$

and

$$
V_{a f}=\left(u+d_{f}(\dot{\alpha}+\dot{\gamma}) \cos (\gamma+\alpha)\right) e_{x}-\left(\dot{z}+d_{f}(\dot{\alpha}+\dot{\gamma}) \sin (\gamma+\alpha)\right) e_{z} .
$$

Furthermore, the hydrodynamic apparent angle of attack of both the extremity of the rudder and the one of the driven foil, which are denoted by $(\beta+\gamma)_{a}$ et $(\alpha+\gamma)_{a}$, are given by the following expressions $\left((.,)_{3}\right.$ is the scalar product in $\left.\mathbb{R}^{3}\right)$ :

$$
\left\{\begin{array}{l}
(\beta+\gamma)_{a}=\operatorname{arsin}\left(\left(e_{y}, \frac{V_{a s}}{\left|V_{a s}\right|} \wedge\left(\cos (\beta+\gamma) e_{x}-\sin (\beta+\gamma) e_{z}\right)_{3}\right)\right. \\
(\alpha+\gamma)_{a}=\operatorname{arsin}\left(\left(e_{y}, \frac{V_{a f}}{\left|V_{a f}\right|} \wedge\left(\cos (\alpha+\gamma) e_{x}-\sin (\alpha+\gamma) e_{z}\right)_{3}\right) .\right.
\end{array}\right.
$$

3.2. The equations of the movement of the boat. The equations of the movement are the following ones (the righthandside is derived from the factor of $\dot{z}$ and $\dot{\gamma}$ in the expression of the power of the hydrodynamical forces):

$$
\left\{\begin{array}{c}
M \ddot{z}-a M \cos (\gamma) \ddot{\gamma}=-M g+\frac{\varrho_{e} S_{s}\left|V_{a s}\right|^{2}}{2} c_{z s}\left((\beta+\gamma)_{a}\right) \\
+\frac{\varrho_{e} S_{f}\left|V_{a f}\right|^{2}}{2} c_{z f}\left((\alpha+\gamma)_{a}\right), \\
-a M \cos (\gamma) \ddot{z}+J_{0} \ddot{\gamma}=-\frac{\varrho_{e} S_{s}\left(h \cos (\gamma)-d_{s} \sin (\gamma)\right)\left|V_{a s}\right|^{2}}{2} c_{z s}\left((\beta+\gamma)_{a s}\right) \\
+\frac{\varrho_{e} S_{f} d_{f} \sin (\alpha+\gamma)\left|V_{a f}\right|^{2}}{2} c_{z f}\left((\alpha+\gamma)_{a f}\right)-M_{0} \\
+\frac{\varrho_{e} S_{s} L\left|V_{a s}\right|^{2}}{2} c_{m s}\left((\beta+\gamma)_{a}\right)+\frac{\varrho_{e} S_{f} L\left|V_{a f}\right|^{2}}{2} c_{m f}\left((\alpha+\gamma)_{a}\right) .
\end{array}\right.
$$


If the point $O$ was chosen as the center of hydrodynamic forces, then one would have $M_{0}=0$.

3.3. On the steady state. First of all, let us characterize the equilibrium position of the ship. The term $\left(\alpha^{0}, \beta^{0}\right)$ corresponding to the equilibrium of the ship over the water $(\gamma=0)$ is solution of:

$$
\left\{\begin{array}{l}
S_{s} c_{z s}\left(\beta^{0}\right)+S_{f} c_{z f}\left(\alpha^{0}\right)=\frac{2 M g}{\varrho_{e} u^{2}}, \\
-\frac{S_{s} h}{L} c_{z s}\left(\beta^{0}\right)+\frac{S_{f} d_{f} \sin \left(\alpha^{0}\right)}{L} c_{z f}\left(\alpha^{0}\right)+S_{s} c_{m s}\left(\beta^{0}\right)+S_{f} c_{m f}\left(\alpha^{0}\right)=\frac{2 M_{0}}{\varrho_{e} L u^{2}} .
\end{array}\right.
$$

It can happen that there is no solution to system (11) and there may be several reasons for this : for instance, it could be because there is no solution at all or because there is no acceptable solution. Indeed, the angles $\alpha^{0}$ and $\beta^{0}$ must be small enough and the interval $\left[-\frac{\pi}{8}, \frac{\pi}{8}\right]$ seems to be reasonable. Or course, in order to solve (11), we have to know the different hydrodynamic coefficients involved in it. We assume in the following computations that these coefficients depend linearly on their variable. It means that the angles $\alpha^{0}$ and $\beta^{0}$ are assumed smaller than the static stall values. We prove the following theorem:

Theorem 3.1. (i) There exists $u_{f}>0$, such that for $u<u_{f}$, system (11) has no solution.

(ii) In case of linear hydrodynamic coefficients, one can choose $u_{f}>0$ such that system (11) has a unique solution for $u \geq u_{f}$ and $u_{f}$ can be explicitely computed.

\section{Proof}

(i) Let us define

$$
G\left(\alpha^{0}, \beta^{0}\right)=\left\{\begin{array}{r}
\frac{\varrho_{e}}{2 M g}\left(S_{s} c_{z s}\left(\beta^{0}\right)+S_{f} c_{z f}\left(\alpha^{0}\right)\right) \\
\frac{\varrho_{e} L}{2 M_{0}}\left(-\frac{S_{s} h}{L} c_{z s}\left(\beta^{0}\right)+\frac{S_{f} d_{f} \sin \left(\alpha^{0}\right)}{L} c_{z f}\left(\alpha^{0}\right)\right. \\
\left.+S_{s} c_{m s}\left(\beta^{0}\right)+S_{f} c_{m f}\left(\alpha^{0}\right)\right)
\end{array}\right.
$$

System (11) can be written as $G\left(\alpha^{0}, \beta^{0}\right)=\frac{1}{u^{2}}\left(\begin{array}{l}1 \\ 1\end{array}\right)$. Let us denote by $\Delta$ the first bisector of $\mathbb{R}^{2}$. System (11) has at leat one solution if and only if

$$
\frac{1}{u^{2}}\left(\begin{array}{l}
1 \\
1
\end{array}\right) \in G\left([0,2 \pi]^{2}\right) \cap \Delta .
$$

The set $G\left([0,2 \pi]^{2}\right) \cap \Delta$ is bounded since $G$ is continuous. Let us set:

$$
U_{0}=\max _{(x, x) \in G\left([0,2 \pi]^{2}\right) \cap \Delta}|x| .
$$

A necessary condition for having solution is:

$$
\frac{1}{u^{2}} \leq U_{0}
$$

and thus $u \geq \frac{1}{\sqrt{U_{0}}}$. The non negative real $u_{f}=\frac{1}{\sqrt{U_{0}}}$ is convenient in order to prove the point (i). 
PH. D.

(ii) We assume that the four hydrodynamic coefficients are linear. Let us write:

$$
\left\{\begin{array}{c}
c_{z s}(\xi)=R_{z s} \xi \\
c_{z f}(\xi)=R_{z f} \xi \\
c_{m s}(\xi)=R_{m s} \xi \\
c_{m f}(\xi)=R_{m s} \xi
\end{array}\right.
$$

By simplifying equation (11), we can easily prove that $\alpha^{0}$ is solution of:

$$
A \alpha^{0}+B \alpha^{0} \sin \left(\alpha^{0}\right)=\frac{C}{u^{2}},
$$

where coefficients $A, B$ et $C$ do not depend on the velocity $u$. Indeed, their values are

$$
\begin{aligned}
A & =\left(\frac{h}{L} S_{f} R_{z f} R_{z s}+S_{f} R_{m f} R_{z s}-S_{f} R_{m f} R_{z f}\right) \\
B & =\frac{h}{L^{2}} S_{f} d_{f} R_{z f} R_{z s}
\end{aligned}
$$

and

$$
C=\frac{1}{\rho_{e}}\left(\frac{2 M g h}{L} R_{z s}+\frac{2 M_{0}}{L} R_{z s}-2 M g R_{m s}\right) .
$$

Let us set $f\left(\alpha^{0}\right)=\frac{1}{C}\left(A \alpha^{0}+B \alpha^{0} \sin \left(\alpha^{0}\right)\right)$. A necessary condition for existence of solution of (13) is that $u$ satisfies:

$$
u \geq \frac{1}{\sqrt{\max _{\left|\alpha^{0}\right| \leq \pi / 8}\left(\left|f\left(\alpha^{0}\right)\right|\right)}} .
$$

Taking into account an analogous condition for the angle $\beta^{0}$, we introduce a critical speed nammed foiling velocity and defined by:

$$
\left.u_{f}=\max \left(\frac{1}{\sqrt{\max _{\left|\alpha^{0}\right| \leq \pi / 8}\left(\left|f\left(\alpha^{0}\right)\right|\right)}}, \min _{\left|\beta^{0}(u)\right| \leq \frac{\pi}{8}}|u|\right)\right) .
$$

The value $u_{f}$ is the minimum speed for which the boat can stand up on the foils. It is obvious that, if $A \neq 0$, the function $f$ is one to one in a neighborhood of 0 . Hence, the best value of $u_{f}$ can be explicited : it depends of all the parameters that appear in $A, B$ and $C$. The proof of (ii) is complete.

The graphs that follow on Figures 6 and 7 provide the function $|f|$ and the steady angles $\alpha^{0}$ et $\beta^{0}$ for $a=0.2$. The foiling speed is nearly $7.2 \mathrm{~m} / \mathrm{s}$ for a $1000 \mathrm{~kg}$ boat which seems reasonable. Of course, the foiling speed depends on the mass of the boat.

3.4. Linearization of the equations. The discussion on the dynamical stability will be organized from a linearization of these equations around the steady angles $\alpha^{0}, \beta^{0}$ solutions of (11). The variables are $z$ and $\gamma$. The first step is to formulate the linearized model around an equilibrium position solution of (11). Let us recall that $\beta$ is assumed to be fixed (equal to $\beta^{0}$ ), and the evolution of the pitching angle of the rear foil is only due to the global pitching 


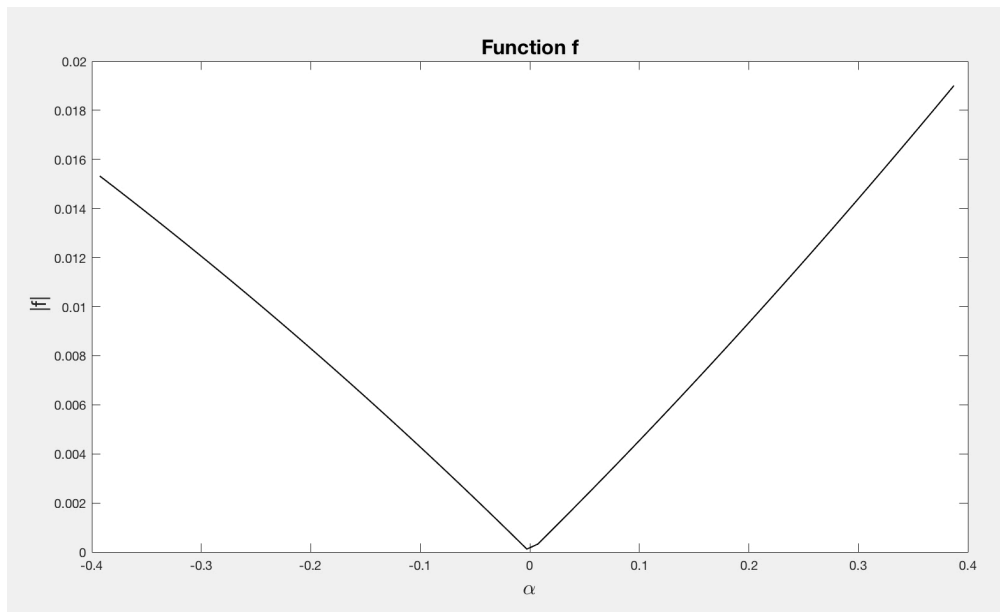

FIGURE 6. Graph of the function $|f|$

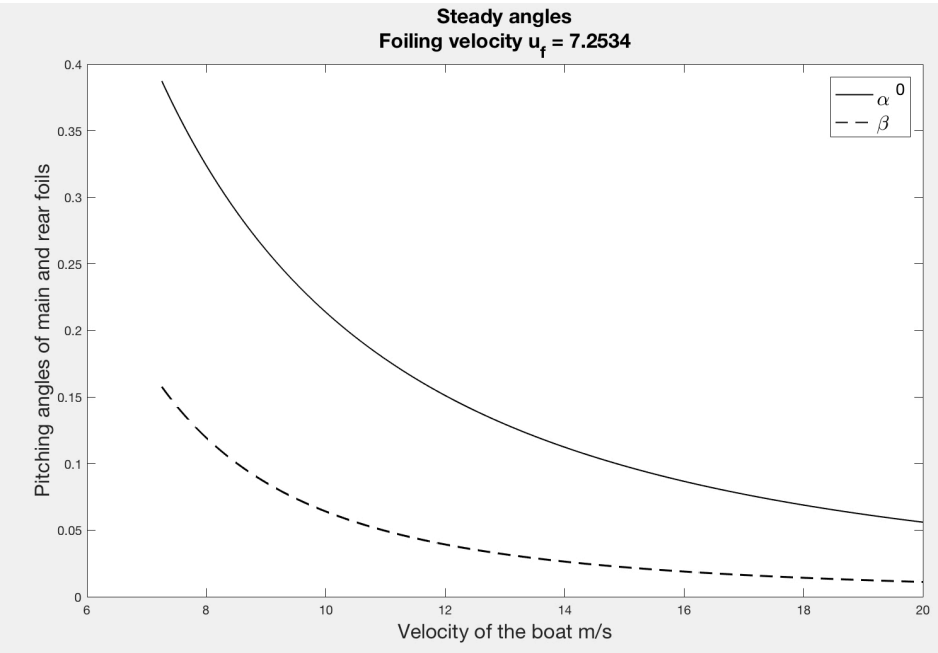

FIGURE 7. The evolution of the steady equilibrium angles

of the boat -say $\gamma$. We write $\delta=\alpha-\alpha^{0}$. In a formal way one can write this linear model as follows:

$$
\left\{\begin{array}{l}
M \ddot{z}-a M \ddot{\gamma}=F_{1}+K_{1} z+T_{1} \gamma+C_{11} \dot{z}+C_{12} \dot{\gamma}+B_{1} \delta+E_{1} \dot{\delta} \\
-a M \ddot{z}+J_{0} \ddot{\gamma}=F_{2}+K_{2} z+T_{2} \gamma+C_{21} \dot{z}+C_{22} \dot{\gamma}+B_{2} \delta+E_{2} \dot{\delta}
\end{array}\right.
$$

In practice, the coefficients $C_{11}, C_{12}, C_{21}$ and $C_{22}$ could be computed using a symbolic computation software if for instance a three dimensional modelling would be concerned. In our two dimensional case it is still possible to perform a hand computation.

We introduce the several matrices of the system - say - $\mathcal{M}, \mathcal{C}$ et $\mathcal{K}$,

$$
\mathcal{M}=\left(\begin{array}{cc}
M & -a M \\
-a M & J_{0}
\end{array}\right), \mathcal{C}=\left(\begin{array}{ll}
C_{11} & C_{12} \\
C_{21} & C_{22}
\end{array}\right), \mathcal{K}=-\left(\begin{array}{ll}
K_{1} & T_{1} \\
K_{2} & T_{2}
\end{array}\right)
$$


and the righthandsides $\mathcal{F}_{0}, \mathcal{B}, \mathcal{E}$ are defined by:

$$
\mathcal{F}_{0}=\left(\begin{array}{c}
F_{1} \\
F_{2}
\end{array}\right), \mathcal{B}=\left(\begin{array}{c}
B_{1} \\
B_{2}
\end{array}\right), \quad \mathcal{E}=\left(\begin{array}{c}
E_{1} \\
E_{2}
\end{array}\right) .
$$

System (15) can now be written: find $X=\left(\begin{array}{l}z \\ \gamma\end{array}\right)$ satisfying

$$
\mathcal{M} \ddot{X}-\mathcal{C} \dot{X}+\mathcal{K} X=\mathcal{F}_{0}+\delta \mathcal{B}+\dot{\delta} \mathcal{E}
$$

with initial data $X(0)=X_{0} \in \mathbb{R}^{2}$ and $\dot{X}(0)=X_{1} \in \mathbb{R}^{2}$. For $\xi_{1}$ and $\xi_{2}$ small enough, we set:

$$
\left\{\begin{array}{c}
c_{z s}\left(\beta^{0}+\xi\right)=c_{z s}^{0}+R_{z s} \xi+o(\xi), \\
c_{z f}\left(\alpha^{0}+\xi\right)=c_{z f}^{0}+R_{z f} \xi+o(\xi), \\
c_{m s}\left(\beta^{0}+\xi\right)=c_{m s}^{0}+R_{m s} \xi+o(\xi), \\
c_{m f}\left(\alpha^{0}+\xi\right)=c_{m f}^{0}+R_{m s} \xi+o(\xi) .
\end{array}\right.
$$

Obviously, we get on the one hand:

and on the other hand:

$$
c_{z s}\left(\beta^{0}\right)=c_{z s}^{0}, c_{z f}\left(\alpha^{0}\right)=c_{z f}^{0}, c_{m s}\left(\beta^{0}\right)=c_{m s}^{0}, c_{m f}\left(\alpha^{0}\right)=c_{m f}^{0},
$$

$$
R_{z s}=c_{m s}^{\prime}\left(\beta^{0}\right), R_{z f}=c_{z f}^{\prime}\left(\alpha^{0}\right), R_{m s}=c_{m s}^{\prime}\left(\beta^{0}\right) \text { et } R_{m f}=c_{m f}^{\prime}\left(\alpha^{0}\right) .
$$

Let us define:

$$
\mathcal{F}(z, \gamma, \dot{z}, \dot{\gamma}, \delta, \dot{\delta})=-M g+\frac{\varrho_{e} S_{s}\left|V_{a s}\right|^{2}}{2} c_{z s}\left((\beta+\gamma)_{a}\right)+\frac{\varrho_{e} S_{f}\left|V_{a f}\right|^{2}}{2} c_{z f}\left((\alpha+\gamma)_{a}\right),
$$

and:

$$
\begin{gathered}
\mathcal{G}(z, \gamma, \dot{z}, \dot{\gamma}, \delta, \dot{\delta})=-\frac{\varrho_{e} S_{s}\left(h \cos (\gamma)-d_{s} \sin (\gamma)\right)\left|V_{a s}\right|^{2}}{2} c_{z s}\left((\beta+\gamma)_{a s}\right)-M_{0}+ \\
+\frac{\varrho_{e} S_{f} d_{f} \sin (\alpha+\gamma)\left|V_{a f}\right|^{2}}{2} c_{z f}\left((\alpha+\gamma)_{a f}\right)+\frac{\varrho_{e} S_{s} L\left|V_{a s}\right|^{2}}{2} c_{m s}\left((\beta+\gamma)_{a}\right)+ \\
+\frac{\varrho_{e} S_{f} L\left|V_{a f}\right|^{2}}{2} c_{m f}\left((\alpha+\gamma)_{a}\right),
\end{gathered}
$$

the righthandsides of the system (10).

The expressions in (15) are (recall that the steady state is oftendenoted by 0) $F_{1}=\mathcal{F}(0)$, $F_{2}=\mathcal{G}(0)$ and partial derivatives of the fonctions $\mathcal{F}$ and $\mathcal{G}$ with respect to $z, \gamma, \dot{z}, \dot{\gamma}, \delta, \dot{\delta}$ at the equilibrium point (in apparition order). With (11), we can assume $\mathcal{F}(0)=\mathcal{G}(0)=0$ thus $F_{1}=F_{2}=0$ and $\mathcal{F}_{0}=0$. The following Lemma is useful for the computation of the derivatives.

Lemma 3.2. We get at the equilibrium point $\left(\alpha^{0}, \beta\right)$ the following expressions:
- $\frac{\partial V_{a f}}{\partial \alpha}=\frac{\partial V_{a f}}{\partial \gamma}=0$
- $\frac{\partial\left|V_{a f}\right|^{2}}{\partial \dot{z}}=0$
- $\frac{\partial}{\partial \dot{z}}\left(\frac{V_{a f}}{\left|V_{a f}\right|}\right)=-\frac{1}{u} e_{z}$
- $\frac{\partial\left|V_{a f}\right|^{2}}{\partial \dot{\gamma}}=2 u d_{f} \cos \left(\alpha^{0}\right)$
- $\frac{\partial\left|V_{a f}\right|}{\partial \dot{\gamma}}=d_{f} \cos \left(\alpha^{0}\right)$
- $\frac{\partial}{\partial \dot{\gamma}}\left(\frac{V_{a f}}{\left|V_{a f}\right|}\right)=-\frac{1}{u} d_{f} \sin \left(\alpha^{0}\right) e_{z}$ 
- $\frac{\partial V_{a s}}{\partial \alpha}(0)=\frac{\partial V_{a s}}{\partial \gamma}(0)=0$

- $\frac{\partial}{\partial \dot{z}}\left(\frac{V_{a s}}{\left|V_{a s}\right|}\right)=-\frac{1}{u} e_{z}$

- $\frac{\partial\left|V_{a s}\right|^{2}}{\partial \dot{\gamma}}=2 u d_{s}$

- $\frac{\partial(\beta+\gamma)_{a}}{\partial \dot{z}}=-\frac{1}{u}$

- $\frac{\partial(\alpha+\gamma)_{a}}{\partial \alpha}=\frac{\partial(\alpha+\gamma)_{a}}{\partial \alpha}=1$

- $\frac{\partial(\alpha+\gamma)_{a}}{\partial \dot{\gamma}}=-\frac{d_{f} \sin \left(\alpha^{0}\right)}{u}$
• $\frac{\partial\left|V_{a s}\right|^{2}}{\partial \dot{z}}=0$

- $\frac{\partial\left|V_{a s}\right|}{\partial \dot{\gamma}}=d_{s}$

- $\frac{\partial}{\partial \dot{\gamma}}\left(\frac{V_{a s}}{\left|V_{a s}\right|}\right)=\frac{h}{u} e_{z}$

- $\frac{\partial(\beta+\gamma)_{a}}{\partial \dot{\gamma}}=\frac{h}{u}$

- $\frac{\partial(\alpha+\gamma)_{a}}{\partial \dot{z}}=-\frac{1}{u}$

The proof, which rests on a simple but long computation, is left to the reader.

3.4.1. Computation of the stiffness matrix $\mathcal{K}$. One obtains:

$$
K_{1}=K_{2}=0 \text { and thus } \mathcal{K}=-\left(\begin{array}{cc}
0 & T_{1} \\
0 & T_{2}
\end{array}\right)
$$

with

$$
\begin{aligned}
& T_{1}=\rho_{e} \frac{u^{2}}{2}\left[S_{s} R_{z s}+S_{f} R_{z f}\right] \\
& T_{2}=\frac{\rho_{e} S_{s}}{2} L u^{2} R_{m s}+\frac{\rho_{e} S_{f}}{2} L u^{2} R_{m f} \\
& +\frac{\rho_{e} S_{f}}{2} d_{f} \cos \left(\alpha^{0}\right) u^{2} c_{z f}^{0}+\frac{\rho_{e} S_{f}}{2} d_{f} \sin \left(\alpha^{0}\right) u^{2} R_{z f} \\
& +\frac{\rho_{e} S_{s}}{2} d_{s} u^{2} c_{z s}^{0}-\frac{\rho_{e} S_{s}}{2} h u^{2} R_{z s} .
\end{aligned}
$$

We introduce the opposite of the dynamical stiffness :

and

$$
R_{1}=\left(\frac{S_{s} R_{z s}}{2}+\frac{S_{f} R_{z f}}{2}\right)
$$

$$
\begin{array}{r}
R_{2}=\frac{1}{2}\left[-S_{s} h R_{z s}+S_{f} d_{f} \sin \left(\alpha_{0}\right) R_{z f}+S_{s} L R_{m s}+S_{f} L R_{m f}\right. \\
\left.+S_{s} d_{s} c_{z s}^{0}+S_{f} d_{f} \cos \left(\alpha_{0}\right) c_{z f}^{0}\right]
\end{array}
$$

The stiffness matrix is then:

$$
\mathcal{K}=-\rho_{e} u^{2}\left(\begin{array}{ll}
0 & R_{1} \\
0 & R_{2}
\end{array}\right)
$$

3.4.2. Computation of the matrix $\mathcal{C}$. The coefficients are given explicitely in the following table: 


\begin{tabular}{|c|c|}
\hline Coefficient & Expression of the coefficients of the matrix $\mathcal{C}$ around $\gamma=0$ \\
\hline$C_{11}$ & $-\frac{\rho_{e}}{2} u\left(S_{s} R_{z s}+S_{f} R_{z f}\right)$ \\
\hline$C_{12}$ & $\rho_{e} u\left(S_{s} d_{s} c_{z s}^{0}+S_{f} d_{f} \cos \left(\alpha^{0}\right) c_{z f}+\frac{S_{s} h}{2} R_{z s}-\frac{S_{f} d_{f}}{2} \sin \left(\alpha^{0}\right) R_{z f}\right)$ \\
\hline$C_{21}$ & $\frac{\rho_{e} u}{2}\left(-S_{s} L R_{m s}-L S_{f} R_{m f}-S_{f} d_{f} \sin \left(\alpha^{0}\right) R_{z f}+S_{s} h R_{z s}\right)$ \\
\hline$C_{22}$ & $-\frac{\varrho_{e} S_{s} h u}{2}\left[2 d_{s} c_{z s}^{0}+h R_{z s}\right]+\frac{\varrho_{e} u S_{f} d_{f}^{2}}{2}\left[\sin \left(2 \alpha^{0}\right) c_{z f}^{0}-\sin \left(\alpha^{0}\right)^{2} R_{z f}\right]$ \\
& $+\frac{\varrho_{e} u L S_{s}}{2}\left[2 d_{s} c_{m s}^{0}+h R_{m s}\right]+\frac{\varrho_{e} u L}{2} S_{f} d_{f}\left[-\sin \left(\alpha^{0}\right) R_{m f}+2 \cos \left(\alpha^{0}\right) c_{m f}^{0}\right]$ \\
\hline
\end{tabular}

Table 2 Expressions of the coefficients $C_{i j}$ around $\gamma=0$ versus $\alpha$ and $\beta$

\section{Stability of the boat.}

4.1. Static stability. We assume in this subsection that the apparent flow velocity is negligible $(\mathcal{C}=0)$, and that the angles of attack at the main and the rear foils are solution of (11) with a velocity larger than the foiling one (it is the one for which the foiling appears). The model is:

$$
\mathcal{M} \ddot{X}+\mathcal{K} X=0 .
$$

Let us set $Y=\left(\begin{array}{c}\dot{X} \\ X\end{array}\right)$. We obtain $Y \in \mathbb{R}^{4}$ and

$$
\dot{Y}=\left(\begin{array}{cc}
\mathcal{O}_{2} & -\mathcal{M}^{-1} \mathcal{K} \\
\mathcal{I}_{2} & \mathcal{O}_{2}
\end{array}\right) Y
$$

We introduce the matrix $4 \times 4 \mathcal{A}$ :

$$
\mathcal{A}=\left(\begin{array}{cc}
\mathcal{O}_{2} & -\mathcal{M}^{-1} \mathcal{K} \\
\mathcal{I}_{2} & \mathcal{O}_{2}
\end{array}\right)
$$

The solutions of

$$
\dot{Y}=\mathcal{A} Y
$$

are $Y(t)=e^{\mathcal{A} t} Y_{0}$ where $Y(0)=Y_{0}$. The stability is governed by the sign of the real part of the eigenvalues of the matrix $\mathcal{A}$ and more precisely the stability is acted if : $(\sigma(\mathcal{A})$ is the spectrum of $A$ )

$$
\forall \lambda \in \sigma(A), \quad \operatorname{Re}(\lambda) \leq 0 .
$$

A vector $v \neq 0$ is an eigenvector of $\mathcal{A}$ if one can find $\lambda \in \mathbb{C}$ such that $\mathcal{A} v=\lambda v$. One can write with $v=\left(\begin{array}{l}v_{1} \\ v_{2}\end{array}\right)$ where $v_{i} \in \mathbb{C}^{2}(\mathrm{i}=1,2)$ :

$$
\left\{\begin{array}{l}
-\mathcal{M}^{-1} \mathcal{K} v_{2}=\lambda v_{1} \\
v_{1}=\lambda v_{2}
\end{array}\right.
$$


or equivalentely: $\left(\lambda^{2} \mathcal{I}_{2}+\mathcal{M}^{-1} \mathcal{K}\right) v_{2}=0$ with $v_{2} \neq 0$. We then deduce that the eigenvalues are also the solutions of

$$
\operatorname{det}\left(\lambda^{2} \mathcal{M}+\mathcal{K}\right)=0
$$

Let us write (recall that $J_{G}=J_{0}-a^{2} M$ ):

$$
\operatorname{det}\left(\lambda^{2} \mathcal{M}+\mathcal{K}\right)=\lambda^{4} M J_{G}-\lambda^{2} \rho_{e} u^{2} M\left(a R_{1}+R_{2}\right) .
$$

The value $\lambda_{0}=0$ is an eigenvalue because of the heaving which is not restricted, and the others one are the solutions of

$$
\lambda^{2}=\frac{\rho_{e} u^{2}}{J_{G}}\left(a R_{1}+R_{2}\right) .
$$

If $a R_{1}+R_{2}>0$, one of them is non negative and therefore an instability (which is coupling between heaving and pitching) may appear. The stability is ensured if :

$$
a R_{1}+R_{2}<0 .
$$

In that case, the solutions are $\lambda= \pm i \mu$ where $\mu$ is the pulsation of the movement and we have:

$$
\mu=u \sqrt{\frac{\varrho_{e}\left|a R_{1}+R_{2}\right|}{J_{G}}} .
$$

The frequency is $f=\frac{\mu}{2 \pi}$. Let us notice that the existence of the instability depends only of $u$ via the angles $\beta^{0}$ and $\alpha^{0}$. There is no direct dependence. For $a$ small enough, the sign of $a R_{1}+R_{2}$ is the same as the sign of $R_{2}$ and is therefore equal to the sign of the trace of the matrix $(\mathcal{K})$ which is non negative. We deduce that the eigenvalues of $\mathcal{K}$ are $\lambda_{1}=0$ and $\lambda_{2}=-\rho_{e} u^{2} R_{2}>0$. If $a=0$, it is easy to check that

$$
J_{0} \ddot{\gamma}+\lambda_{2} \gamma=0 \text {. }
$$

Therefore

$$
\gamma(t)=\gamma_{0} \cos \left(\sqrt{\frac{\lambda_{2}}{J_{0}}} t\right)+\frac{\gamma_{1} \sqrt{J_{0}}}{\sqrt{\lambda_{2}}} \sin \left(\sqrt{\frac{\lambda_{2}}{J_{0}}} t\right) .
$$

Since $\ddot{z}=-\frac{\lambda_{2}}{M} \gamma$, and since the initial data must satisfy (11), one can see that the angle of attack of the main foil $\alpha^{0}$ must be adjusted in order that the boat resists capsizing.

The Figures 8 and ?? illustrate the numerical computation of the eigenvalues when the hydrodynamics coefficients linearly depend of their variables for sake of simplicity. The velocity $u$ begins at $1 \mathrm{~m} / \mathrm{s}$ and up to $10 \mathrm{~m} / \mathrm{s}$ with 300 steps in time. At each iteration, the values of the angles $\beta^{0}$ and $\alpha^{0}$ at the equilibrium are computed with the formulae (11). Once computed, the spectrum of the matrix $\mathcal{A}$ is obtained. We have drawn the real part (damping or increase) and the frequency of the movement $f$ with respect to the velocity $u$. We can notice an instability in heaving due to the double null eigenvalue. The pitching is stable since 0 is the only real eigenvalue. Moreover, $a>0$ means that the center of mass of the boat is behind (but not far from ) the point $O$. We have drawn the graphs for $a=0.2$ and $a=0.8$ with $h=5$. The frequencies seems to be near $1 \mathrm{~Hz}$ or $1.5 \mathrm{~Hz}$ for large values of $u$. There is an instable region for small speeds for $a=0.8 \mathrm{~m}$.

The Figures 10 presents the same graphs for $h=7$. One can notice that the movement is stable for both values of $a=0.2$ and $a=0.8$.

The Figures 11 and 12 for different hydrodynamics coefficients, smaller at point $S$ than at point $F$, for $h=5$. Same graphs for $h=7$ concern the Figure ?? and Figure ??.

The Figures 13 and 14 concern the previous phenomenon. On Figure 13, we have drawn $R_{2}+a R_{1}$ with respect to $a$ and $u$ (recall that the stability is ensured if $R_{2}+a R_{1}<0$ ). 
PH. D.
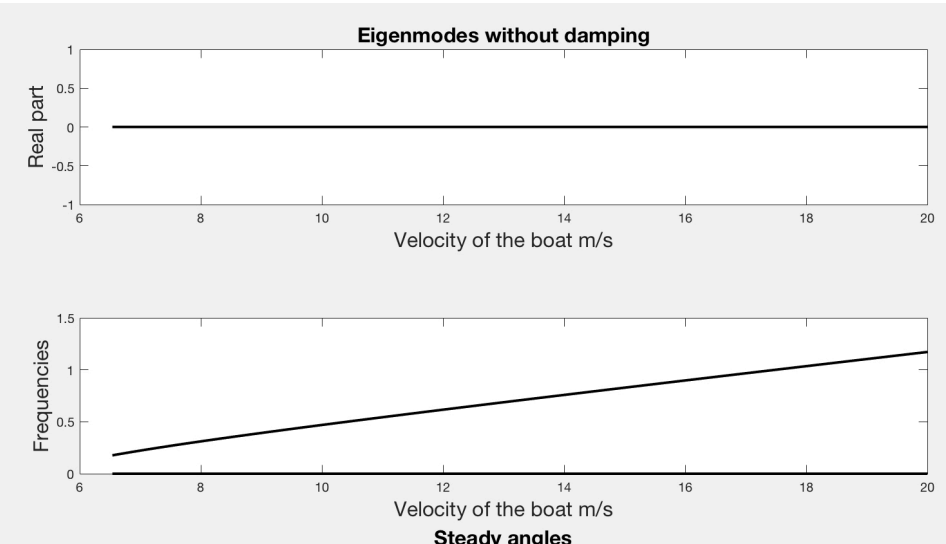

Steady angles

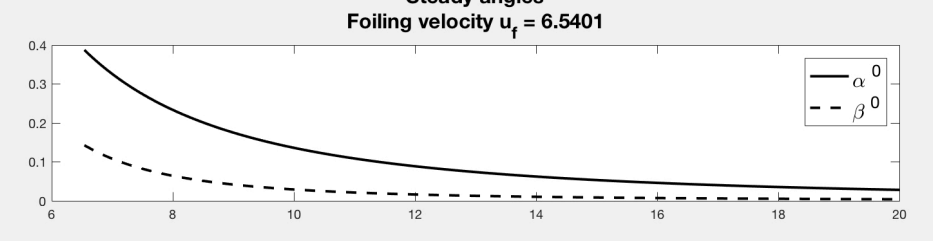

FIGURE 8 . The evolution of the spectrum versus the velocity of the boat with $a=0.2 \mathrm{~m}$ and $h=5 \mathrm{~m}$

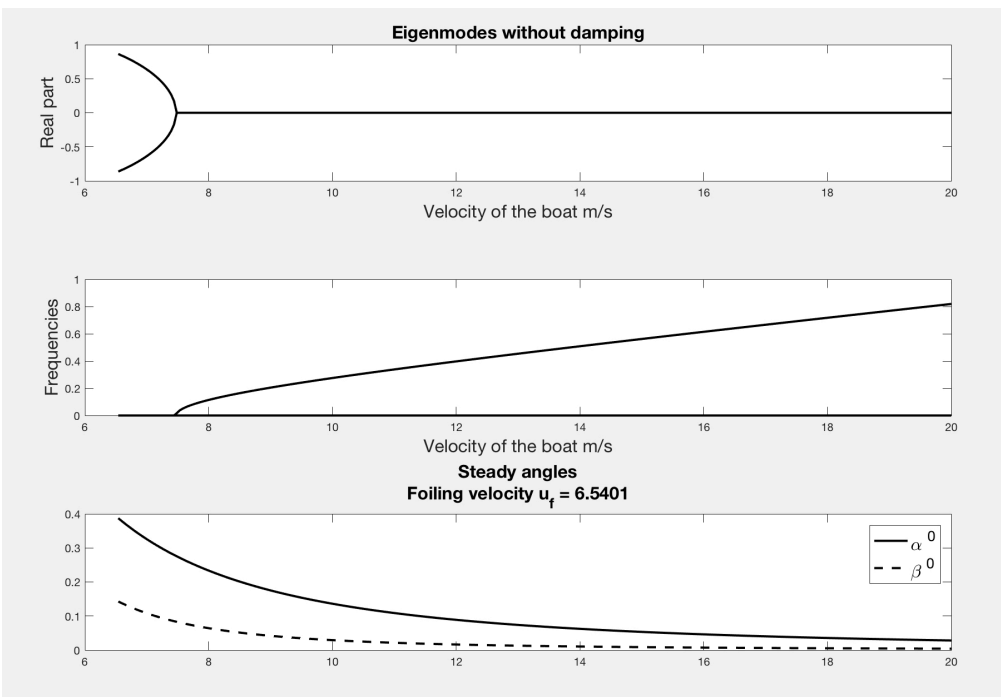

FIGURE 9. The evolution of the spectrum versus the velocity of the boat with $a=0.8 \mathrm{~m}$ and $h=5 \mathrm{~m}$

One can notice that for large value of the parameter $a$ (the distance between the center of mass and the daggerboad), there is a critical value $u_{c}=u_{c}(a)$ under which instability may occur. The Figure 14 is the graph of $u_{c}$ with respect to $a$. These graphs are achieved with $h=5$. The Figures 15 and 16 illustrate the same phenomenon in the case of different hydrodynamics coefficients. 

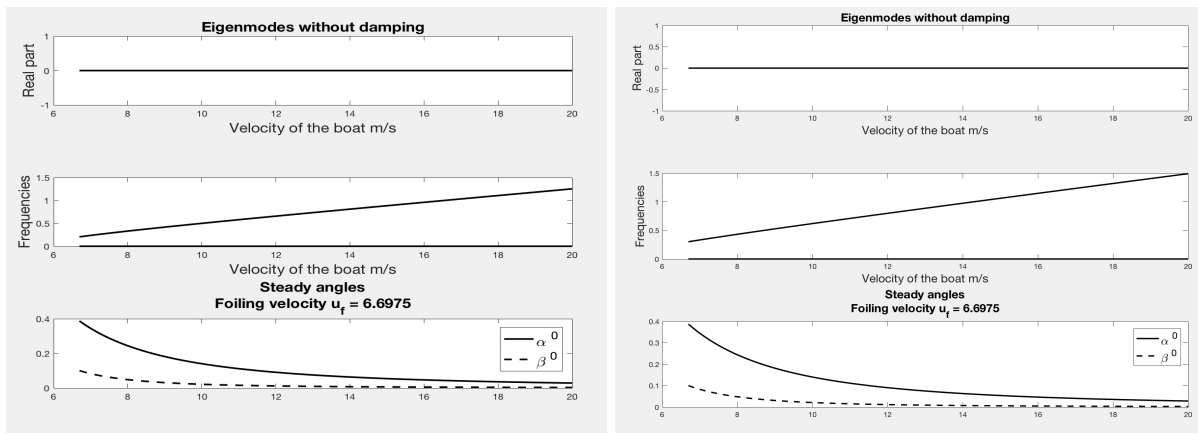

FIGURE 10. The evolution of the spectrum versus the velocity of the boat with $a=0.8 \mathrm{~m}$ (left) and $a=0.2 \mathrm{~m}$ (right) with $h=7 \mathrm{~m}$
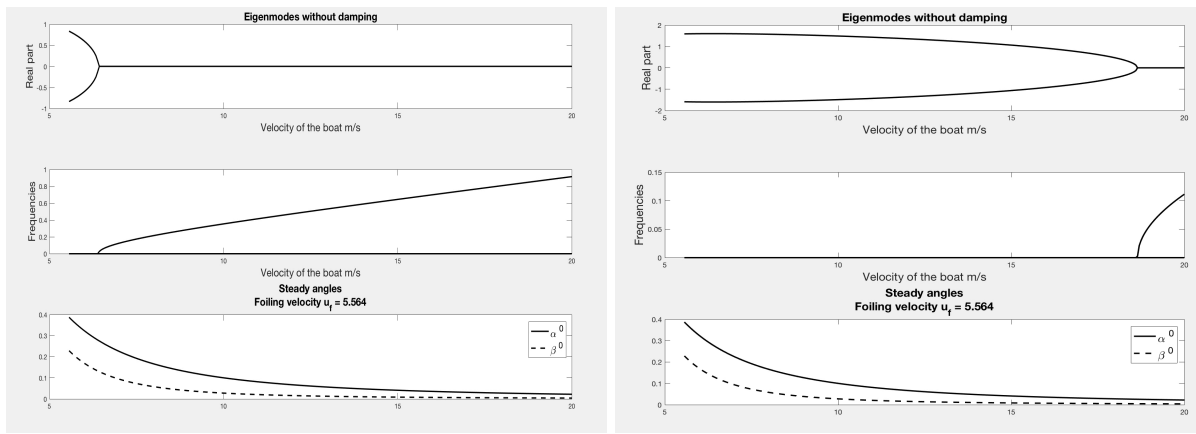

FIGURE 11. The evolution of the spectrum versus the velocity of the boat with $a=0.2 \mathrm{~m}$ (left) and $a=0.8 \mathrm{~m}$ (right), $h=5 \mathrm{~m}$ and different hydrodynamics coefficients
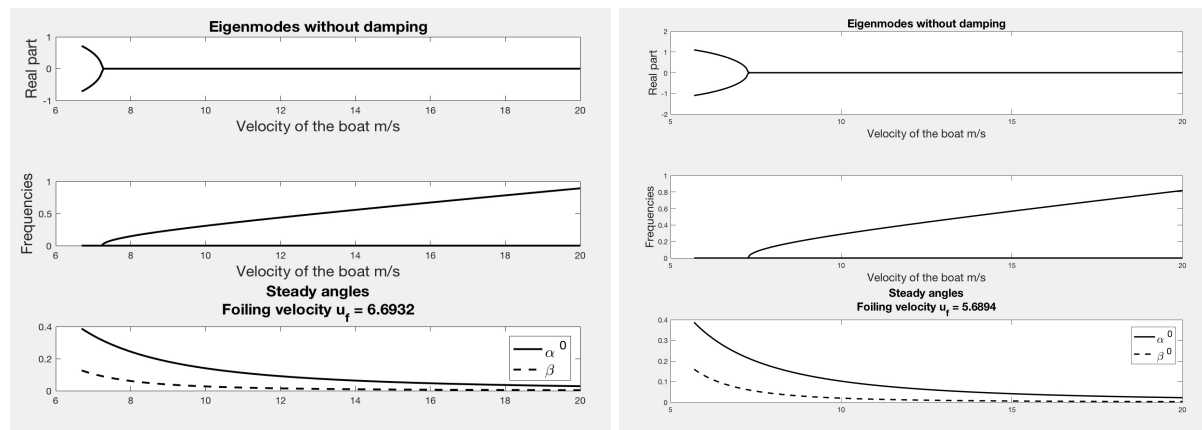

FIGURE 12. The evolution of the spectrum versus the velocity of the boat with $a=1 \mathrm{~m}$ (left) and $a=0.8 \mathrm{~m}$ (right) with $h=7 \mathrm{~m}$ and different hydrodynamics coefficients

4.2. Dynamic stability. Let us notice that the dynamical behavior of a single foil is presented in [8] where the hydroelastic response and stability of both rigid and flexible 2D hydrofoils in viscous flow is discussed from experimental and numerical aspects. There are in general four kinds of dynamic instabilities which can occur in general. 


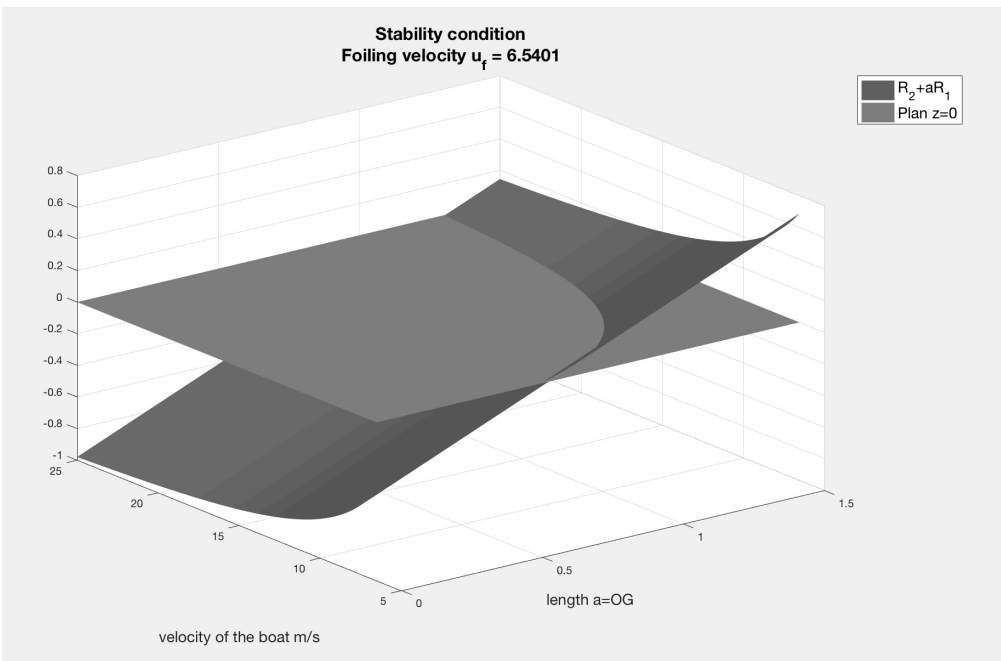

FIGURE 13. The criterium of stability with respect to $a$ and $u$

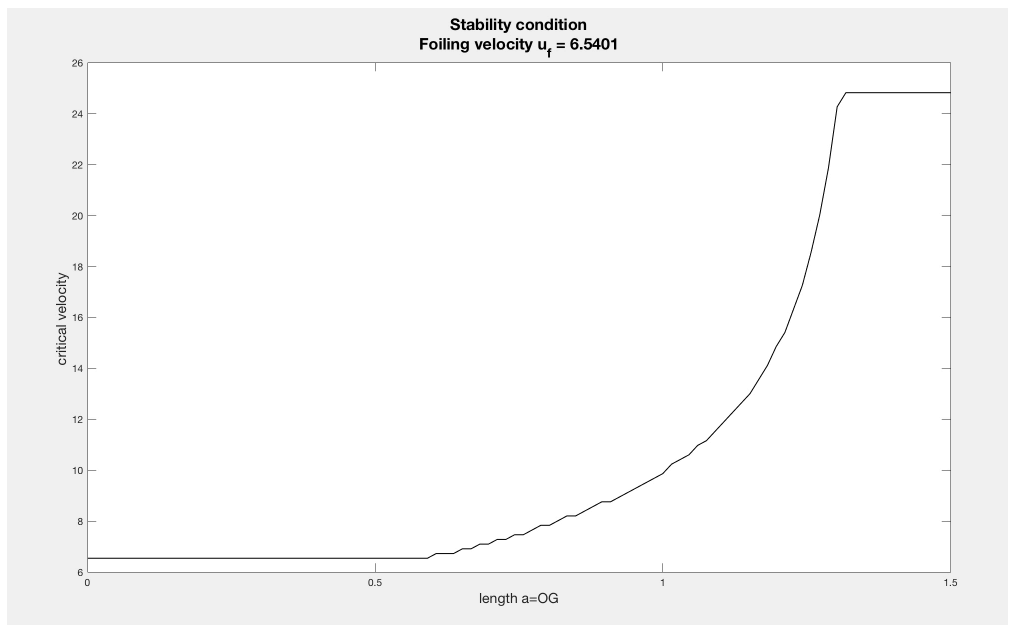

FIGURE 14 . The critical velocity with respect to $a$ is sensitive to small value of $a$

1. One is well known by the sailors. It concerns induced vibrations on the rudder due to vortices created by the main foil. But, this so called buffting effect (see [5] [9]), can occur only for very particular cases. In our case it would be due to a vortex shedding from the main foil onto the one of the rudder. And it would appear if the frequency of the vortices is close to the one of the rudder and its foil. It is quickly detected and should be suppressed by an ad'hoc conception of the ship. It is not necessarily destroying but can reduce considerably the efficiency as far as it takes energy from the kinetical energy of the boat.

2. The second one is the classical flutter which is violent and corresponds usually to the unlimited exchange of energy between two movements with the same eigenfrequency (here for the heaving and the pitching). Clearly the secure flight of a ship would be 


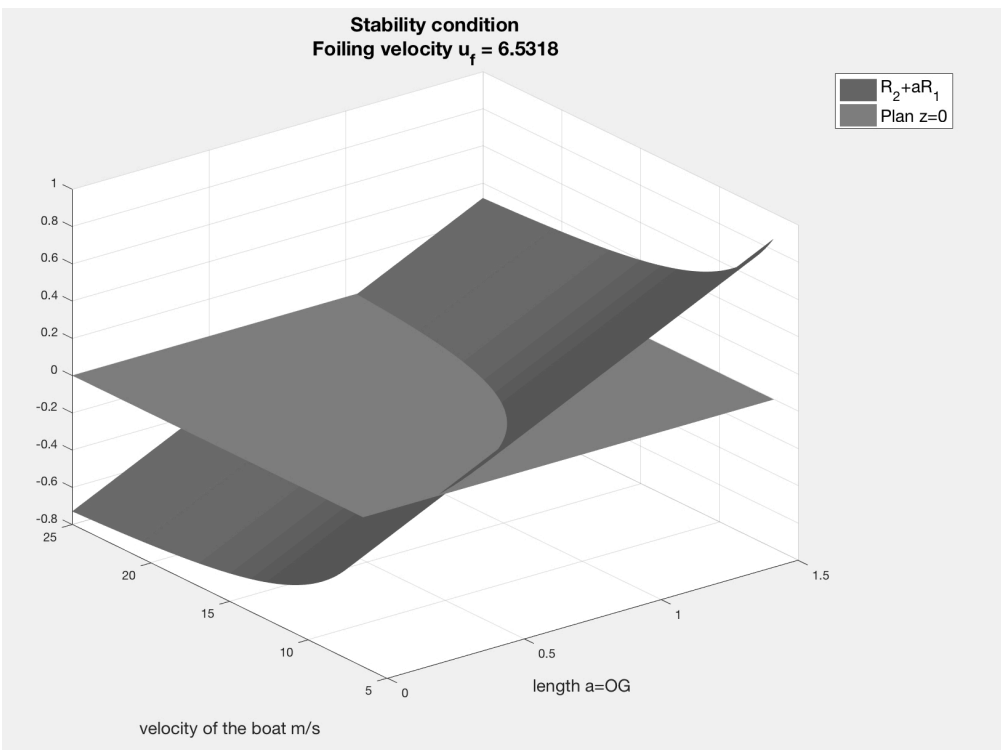

FIGURE 15. The criterium of stability with respect to $a$ and $u$

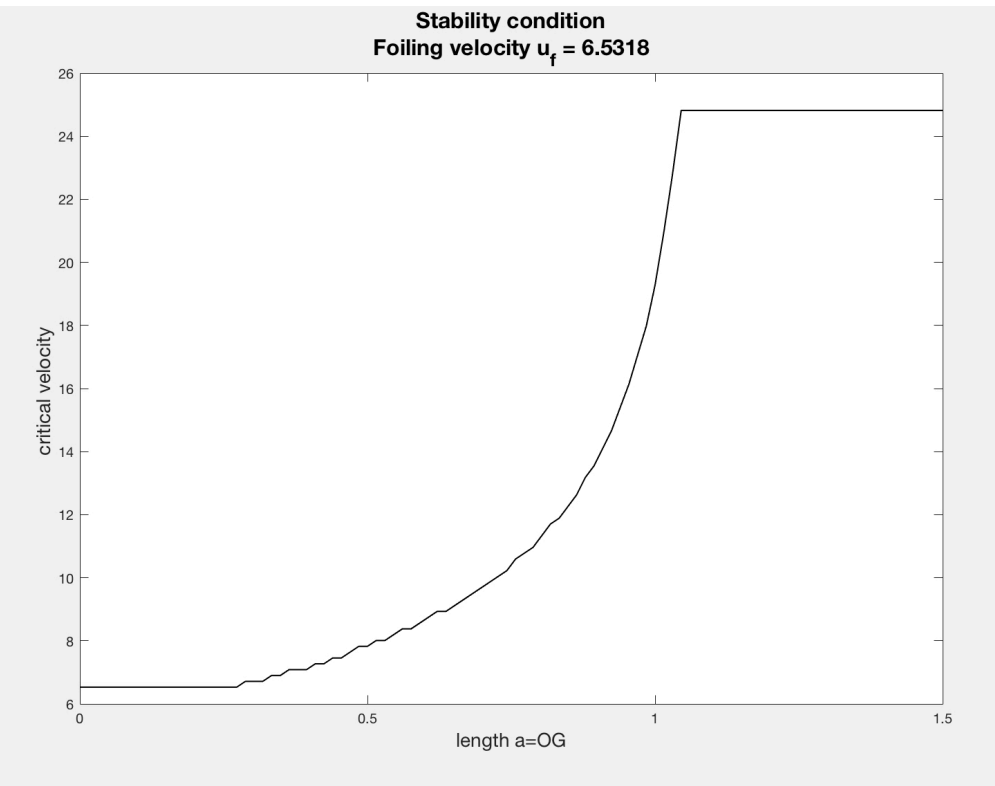

FIGURE 16. The critical velocity with respect to $a$ is sensitive to small value of $a$

seriously compromised by such an instability implying an exponential increase of the movement of the ship [9]. It would be difficult to control it using the main foil without additional lifting supplementary wing. Furthermore, because the phenomenon is very quick and complex, its control requires an automatic loop driven by an electronic computer. In fact as far as the boat is flying over the water, there no stiffness on the 
heaving excepted the one due to the hydrodynamical forces acting on the foils which are fully immersed in our model. May be it would be different if the bows were in contact with the water and the Archimède forces would operate.

3. The third possibility of instability is due to a fluctuation of the wind velocity. It is a rather complex phenomena implying the apparent flow velocity but mainly the perturbation of the flow due to vortex shedding. The rudder is the part mainly concerned by this phenomenon due to the turbulence generated from the main foil. It is under the skipper/helmsman control. It is discussed in section 2.2. It could be compared from the mathematical point of view to the buffting phenomenon.

4. The fourth dynamical instability and the last one in our discussion, is due to the apparent water velocity on the foils. It could be compared to a stall flutter phenomenon as the one encountered in the breakdown of the famous Tacoma-Narrows bridge which collapsed on November 1940. This accident was correctly explained forty years later by R. Scanlan [11] and the final explaination rests on the apparent flow velocity. From the mechanical point of view, the phenomenon can be understand as a negative damping. See also [6] for a similar collapse of a model of a military aircraft.

We are mainly interested in this paper in the case of stall flutter phenomenon (case 4). We then consider the model:

$$
\mathcal{M} \ddot{X}-\mathcal{C} \dot{X}+\mathcal{K} X=0 .
$$

We still set $Y=\left(\begin{array}{l}\dot{X} \\ X\end{array}\right)$. We have $Y \in \mathbb{R}^{4}$ and :

$$
\dot{Y}=\left(\begin{array}{cc}
\mathcal{M}^{-1} \mathcal{C} & -\mathcal{M}^{-1} \mathcal{K} \\
\mathcal{I}_{2} & \mathcal{O}_{2}
\end{array}\right) Y
$$

Following the previous section, we have to consider the spectrum of the following matrix $\mathcal{A}$ :

$$
\mathcal{A}=\left(\begin{array}{cc}
\mathcal{M}^{-1} \mathcal{C} & -\mathcal{M}^{-1} \mathcal{K} \\
\mathcal{I}_{2} & \mathcal{O}_{2}
\end{array}\right)
$$

Let $v \neq 0$ be an eigenvector of $\mathcal{A}$. One has $\mathcal{A} v=\lambda v(\lambda \in \mathbb{C})$ and with $v=\left(\begin{array}{l}v_{1} \\ v_{2}\end{array}\right)$ with $v_{i} \in \mathbb{C}^{2}(\mathrm{i}=1,2)$ :

$$
\left\{\begin{array}{l}
\mathcal{M}^{-1} \mathcal{C} v_{1}-\mathcal{M}^{-1} \mathcal{K} v_{2}=\lambda v_{1} \\
v_{1}=\lambda v_{2}
\end{array}\right.
$$

which leads to

$$
\left(\lambda^{2} \mathcal{I}_{2}-\lambda \mathcal{M}^{-1} \mathcal{C}+\mathcal{M}^{-1} \mathcal{K}\right) v_{2}=0
$$

with $v_{2} \neq 0$. Therefore

$$
\operatorname{det}\left(\lambda^{2} \mathcal{M}-\lambda \mathcal{C}+\mathcal{K}\right)=0,
$$

which is an equation like $\lambda\left(A \lambda^{3}+B \lambda+C\right)=0$. The stability of (23) is ensured if the solutions are simple and with a negative real part. We have $\lambda=0$ or:

$$
\begin{gathered}
\lambda^{3} m J_{G}-\lambda^{2}\left(m c_{22}+c_{11} J_{0}+a\left(c_{21}+c_{12}\right)\right)+\lambda\left(c_{11} c_{22}-c_{21} c_{12}-\rho_{e} m u^{2}\left(R_{2}+a R_{1}\right)\right) \\
+\rho_{e} u^{2}\left(c_{11} R_{2}-c_{21} R_{1}\right)=0 .
\end{gathered}
$$

If one consider the pulsation, then $\lambda=i \mu$ and $\mu$ is solution of

$$
\operatorname{det}\left(-\mu^{2} \mathcal{M}-i \mu \mathcal{C}+\mathcal{K}\right)=0,
$$

and the stability condition becomes $\operatorname{Im}(\mu)>0$. 
The Figures 17 concerns the dynamic stability. On the upper graph of the Figure 17(left), we have drawn the maximum and minimum of the real part of the spectrum. On the centered graph, we have drawn the frequencies, and the down graph is the one of the equilibrium angles. On the Figure 17 (right), we have drawn the maximum of the real part of the spectrum $\mathcal{A}$ with respect to the speed. The parameters are: $a=1 \mathrm{~m}$ and $h=5 \mathrm{~m}$. The Figures 18 concerns the case $a=0.2 \mathrm{~m}$ et $h=5 \mathrm{~m}$. On Figures 22, 23 and 24, the criterion of stability is drawn with respect to $a$ and $u$ respectively in case of identical hydrodynamic coefficients for $h=5 \mathrm{~m}, h=7 \mathrm{~m}$ and different hydrodynamic coefficents $\left(c_{z s}\right.$ and $\left.c_{z f}\right)$ : the stability is ensured under the plan $z=0$.
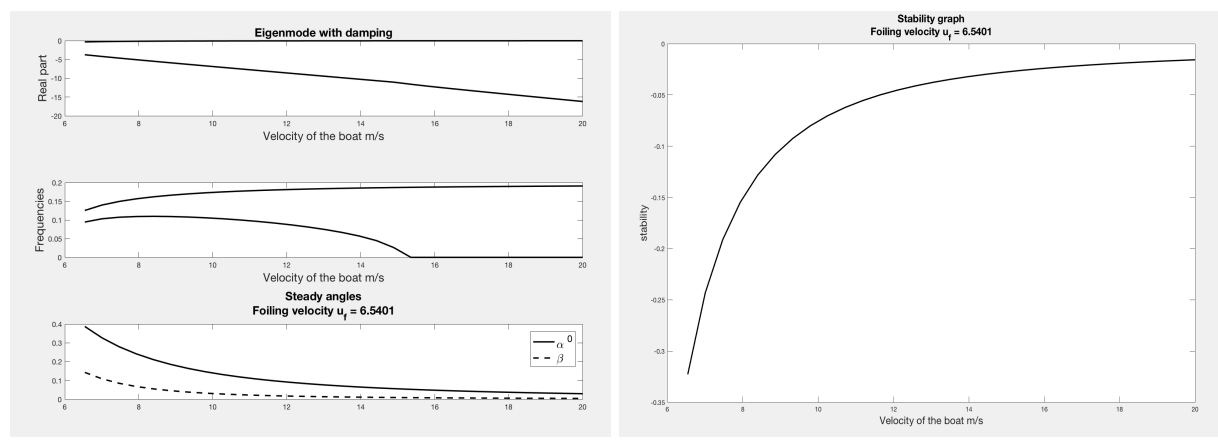

FIGURE 17. The spectrum (left) and criterium of stability (right) for $a=$ 1 and $h=5$
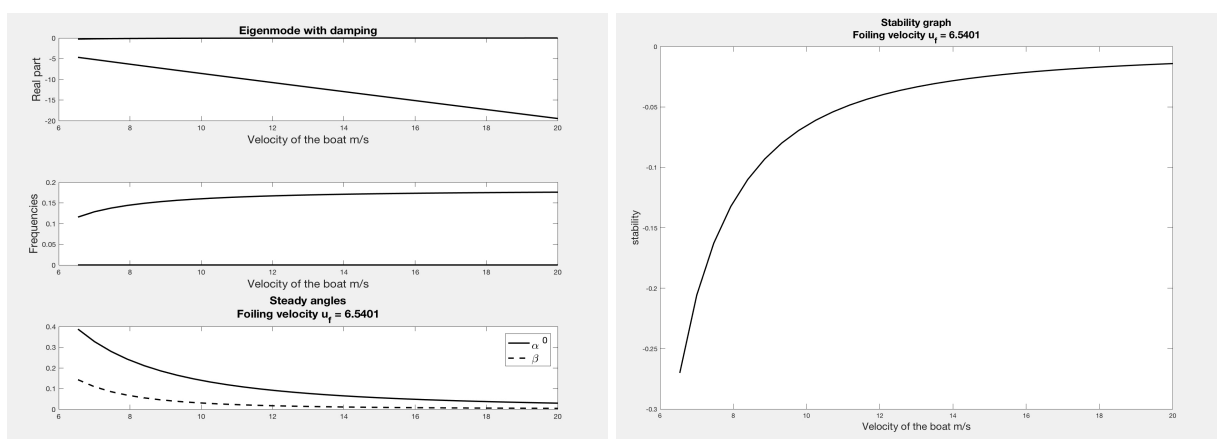

FIGURE 18. The spectrum (left) and criterium of stability (right) for $a=$ 0.2 and $h=5$.

The Figure 20 and 19 concern the case of different hydrodynamic coefficients for decreasing value of the parameter $a$ and $h=5$. As shown on Figure 19, the stability is ensured but one can see that the eigenmodes are quite differents.

The Figure 21 concerns the case of different hydrodynamic coefficients for $a$ and $h=7$. Same phenomena occur.

\section{References}

[1] R. Bellman [1957], Dynamic programming, Dover edition.

[2] H. Brezis, "Analyse Fonctionnelle," edition Masson, Paris, 1983.

[3] Jean Cea [1968], Optimisation, Théorie et Algorithmes, Dunod, Paris.

[4] P.-G. Ciarlet [1985], Introduction à l'analyse numérique et à l'optimisation, Masson, Paris. 
PH. D.
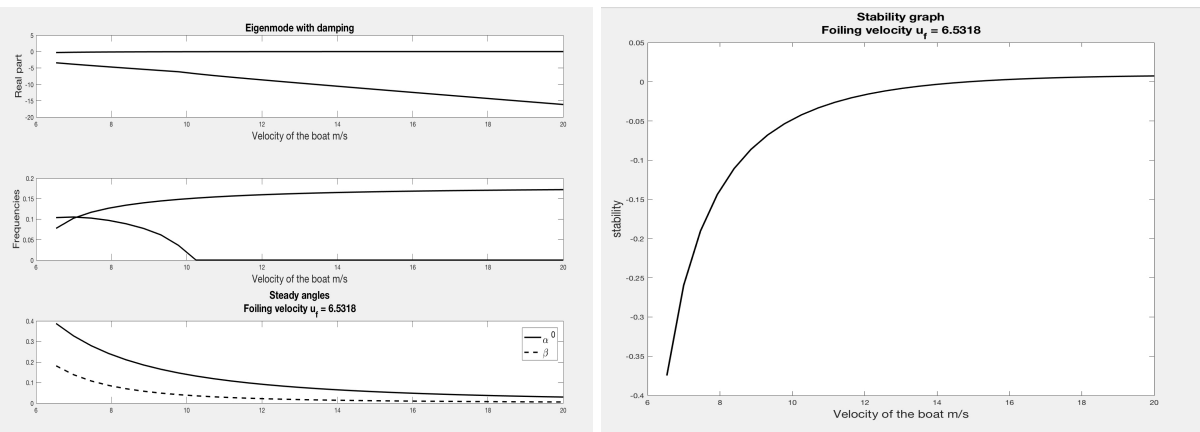

FIGURE 19. The spectrum (left) and criterium of stability (right) for $a=0.2 \mathrm{~m}$
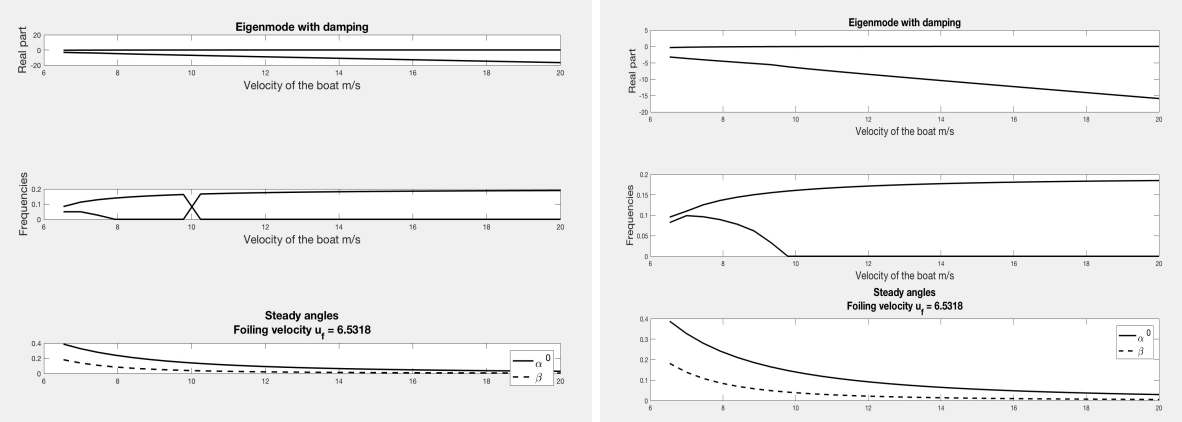

FIGURE 20. The spectrum for $a=1 \mathrm{~m}$ and $a=0.8 \mathrm{~m}:$ stable in both cases
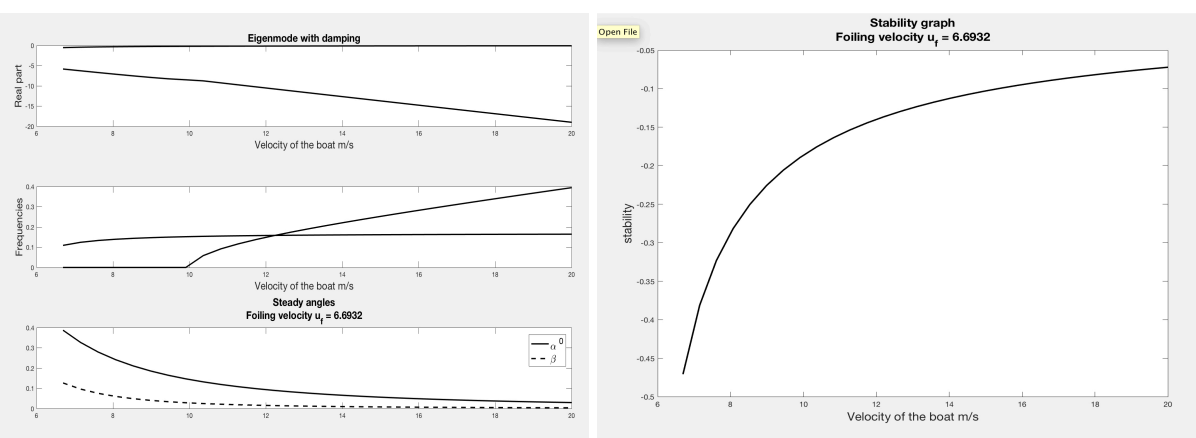

FIGURE 21. The spectrum (left) for $a=1 \mathrm{~m}$ and criterium of stability (right)

[5] Ph. Destuynder [2007], Introduction à l'aéroélasticité et à l'aéroacoustique. Hermès-Lavoisier, ParisLondres.

[6] Ph. Destuynder [2010], Analyse et contrôle des équations différentielles, Hermès-Lavoisier, Paris-Londres.

[7] $\mathrm{Ph}$. Destuynder and M. T. Ribereau [1996], Non linear dynamics of test models in wind tunnels, in Eur. J. Mech. A/Solids, $15, n^{\circ} 1$, p. 91-136.

[8] A. Ducoin and Y.-L. Young [2013], Hydroelastic response and stability of a hydrofoil in viscous flow, in Journ. of Fluids and Structures, (38), p. 40-57, 2013.

[9] Y.C. Fung, [1969], An introduction to the theory of aeroelasticity, Dover publication.

[10] J.L. Lions, [1988], Contrôlabilité exacte, perturbations et stabilisation de systèmes distribués, Masson, Paris. 


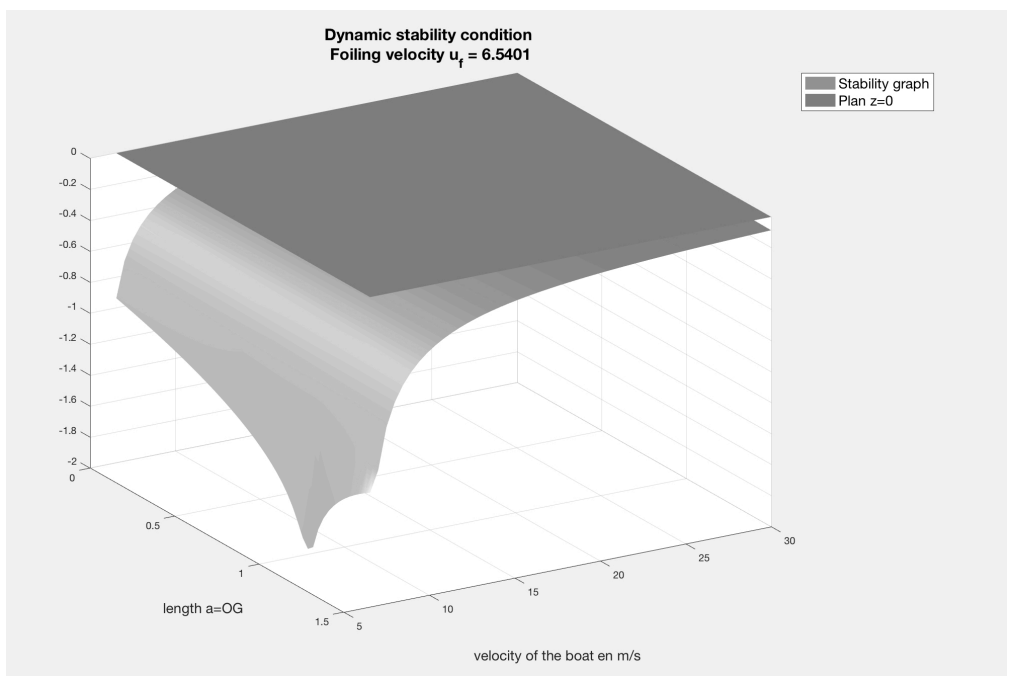

FIGURE 22. The criterium of stability with respect to $a$ and $u$ for $h=5$

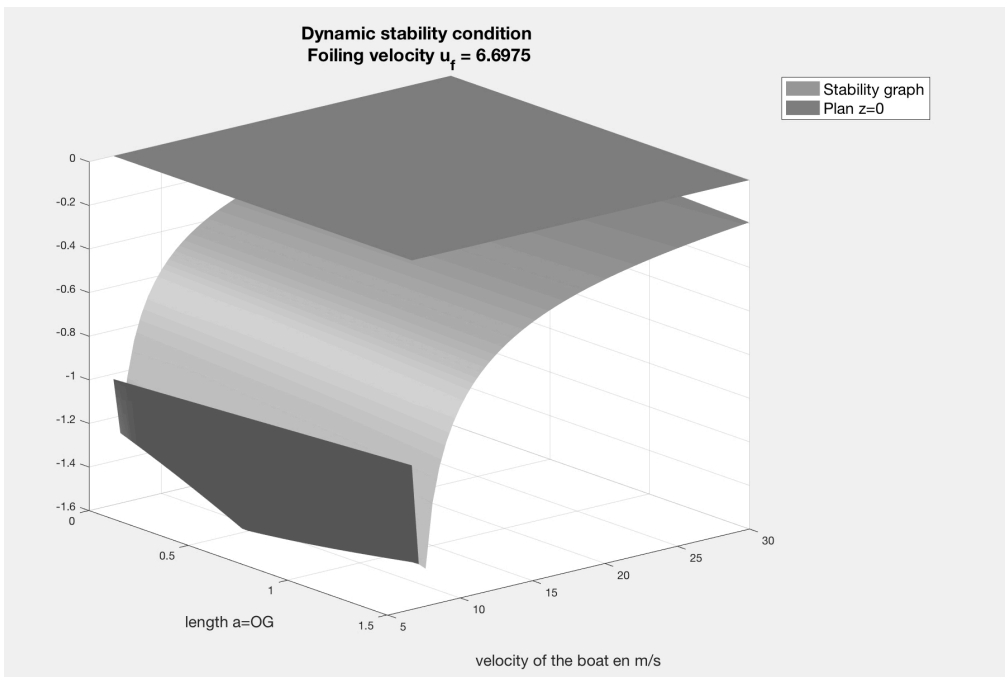

FIGURE 23. The criterium of stability with respect to $a$ and $u$ for $h=7$

[11] E.H. Dowell, H.C. Curtiss Jr., R.H. Scanlan, F. Sisto [1978], A Modern Course in Aeroelasticity. Monographs and textbooks of solids and fluids. Alphen aan den Rijn, Sijthoff and Noordhoff International Publishers.

[12] A.J Hermans, G.C Hsiao and R. Timman, Water waves and ship hydrodynamics, Delft University Press, The Netherlands, (1985) 
PH. D.

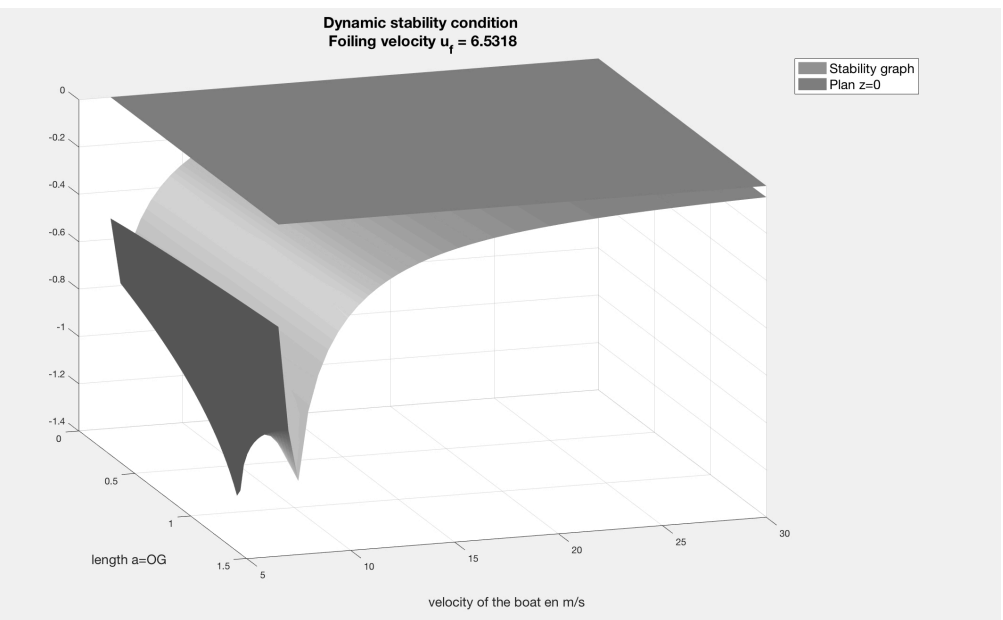

FIGURE 24. The criterium of stability in case of different values of the hydrodynamic coefficients 